AIAA Paper No. 86-0136

\title{
SPRAY ATOMIZATION AND COMBUSTION
}

\author{
G. M. Faeth* \\ Aerospace Engineering Department \\ The University of Michigan \\ Ann Arbor, Michigan 48109-2140
}

\begin{abstract}
Recent advances concerning the analysis of sprays and drop/turbulence interactions are reviewed. Consideration is given to both dilute sprays which contain more-or-less spherical drops and have liquid fractions less than 1 percent; and dense sprays, which comprise nearinjector conditions with irregularly-shaped liquid elements and relatively high liquid fractions.

Early analysis of dilute sprays assumed either locally-homogeneous flow (LHF), implying infinitely-fast interphase transport rates, or deterministic separated flow (DSF) where finite interphase transport rates are considered in the mean but drop/turbulence interactions are ignored. These limits are useful in some instances, however, recent evidence shows that both methods are deficient for quantitative estimates of the structure of practical sprays. As a result, stochastic separated flow (SSF) methods have been developed which treat both finite interphase transport rates and drop/turbulence interactions using random-walk computations for drop properties. Evaluation of SSF a' 'sis of particle-laden jets; nonevaporating, evaporating arvombusting sprays; and noncondensing and condensing bubbly jets has been encouraging. However, many fundamental problems must still be resolved for dilute sprays, e.g., effects of anisotropic turbulence, modification of continuous-phase turbulence properties by the dispersed phase, effects of turbulence on interphase transport rates, and drop shattering, among others.
\end{abstract}

Dense spray processes are poorly understood due to substantial theoretical and experimental difficulties, e.g., the idealization of spherical dispersed-phase elements is unrealistic, important effects of liquid breakup and collisions are difficult to describe, spatial resolution is limited, and the flow is opaque to laser-based instruments which have been helpful for studies of dilute sprays. Limited progress thus far, however, suggests that LHF analysis may provide a useful first step toward quantifying the structure and mixing processes of dense sprays near pressure atomizing injectors.

\section{Nomenclature}
a acceleration of gravity
C particle concentration
$C_{D}$ drag coefficient
$C_{i}$ parameters in turbulence model
$C_{P}$ specific heat at constant pressure
d injector diameter
d. particle diameter
coefficient of mass diffusion

*A.B. Modine Professor of Aerospace Enginecring, Associate Fellow AIAA.

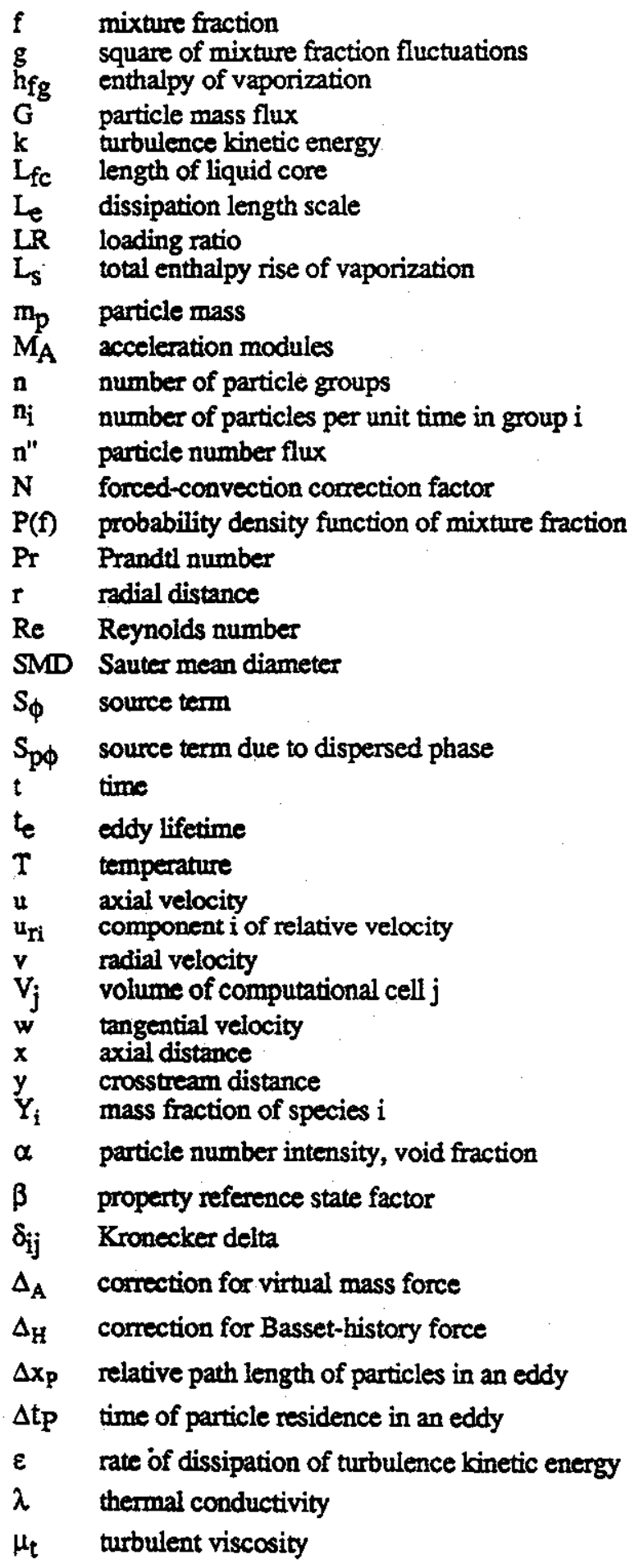



variable of integration

$\rho$ density

$\sigma_{i} \quad$ turbulent Prandt $/$ Schmidt number

$\phi \quad$ generic property

\section{Subscripts}

$\begin{array}{ll}\text { c } & \text { centerline property } \\ \text { f } & \text { liquid property } \\ \text { F } & \text { evaporating fluid } \\ \text { p } & \text { particle property } \\ \text { ref } & \text { reference state } \\ \text { s } & \text { particle surface property } \\ \text { o } & \text { injector exit condition } \\ \infty & \text { ambient condition }\end{array}$

\section{Superscripts}

(一), ( )' time-averaged mean and fluctuating quantity $(\sim),()^{\prime \prime} \quad$ Favre-averaged mean and fluctuating quantity $\rightarrow$ vector quantity

\section{Introduction}

In spite of their importance, our understanding of sprays is relatively limited since interactions between phases must be resolved along with the usual problems of analyzing turbulence. There has been progress in the field, however, due to the emergence of new theoretical and experimental methods for treating sprays. The objective of this paper is to review these findings and to suggest areas where additional research is needed.

Aspects of sprays have been considered in several reviews. Soo (1967) presents a comprehensive treatment of early work. Particle-laden flows which are closely related to sprays, are considered by Hinze (1972,1975), Goldschmidt et al. (1972) and Peskin (1975). Interphase transport properties of drops, emphasizing nonturbulent effects, are considered by Faeth (1977), Clift et al. (1978), Law (1982) and Sirignano (1983). Finally, Bracco (1983,1985), Chigier (1976), Crowe (1982), Faeth (1983a,b), Lefebvre (1980) and Williams (1985) review various aspects of spray structure. The present paper is an extension of Faeth $(1983 a, b)$, discussing recent work on spray analysis and its evaluation with measurements.

Sprays and other dispersed flows are normally divided into dilute and dense flow regimes. Both regimes are considered in this review. Dilute sprays contain moreor-less spherical drops and have relatively small liquid volume fractions (generally less than 1-10 percent). As a result, drop collisions are infrequent and interphase transport rates are not directly influenced by adjacent drops. This region does not correspond to the behavior of isolated drops in a known environment, however, since drop transport in dilute sprays influences the structure of the continuous phase. Our understanding of dilute sprays has developed rapidly in the past decades due to the emergence of optical diagnostics and new methods for analyzing turbulence. Major unresolved issues for dilute sprays involve drop/turbulence interactions, e.g., the turbulent dispersion of drops, the modification of continuous-phase turbulence properties by drops (nurbulence modulation), and the effect of turbulence on interphase transport rates.

The dense-spray regime near the injector exit involves the transition between an all-liquid flow in a passage and the dilute dispersion of drops which marks the start of the dilute spray regime. In addition to large liquid fractions, dense sprays are characterized by irregularlyshaped (as opposed to spherical) liquid elements. Phenomena which are complex to analyze -- effects of collisions between liquid elements, breakup of liquid elements, and direct effects of nearly elements on interphase transport rates - are all important in dense sprays. In addition, measurements are difficult in dense sprays due to the need for high spatial resolution and the opacity of the flow to optical diagnostics. Available information on dense sprays is discussed in the following, along with suggestions for circumventing some of the difficulties.

The paper begins with a description of common methods used to analyze dilute sprays. These procedures are then used to interpret recent measurements of the structure of dilute sprays and related dispersed turbulent jets. The following flows are considered: particle-laden jets; nonevaporating, evaporating and combusting sprays; and noncondensing and condensing bubbly jets. The actual data base is summarized in Table 1. These flows also serve as an initial evaluation of current methods of analysis for a wide range of conditions. Work considered by the author and his associates is emphasized for dilute sprays since it provides a common basis of analytical and experimental methods. The paper concludes with a brief discussion of dense sprays, where existing information largely results from the work of others.

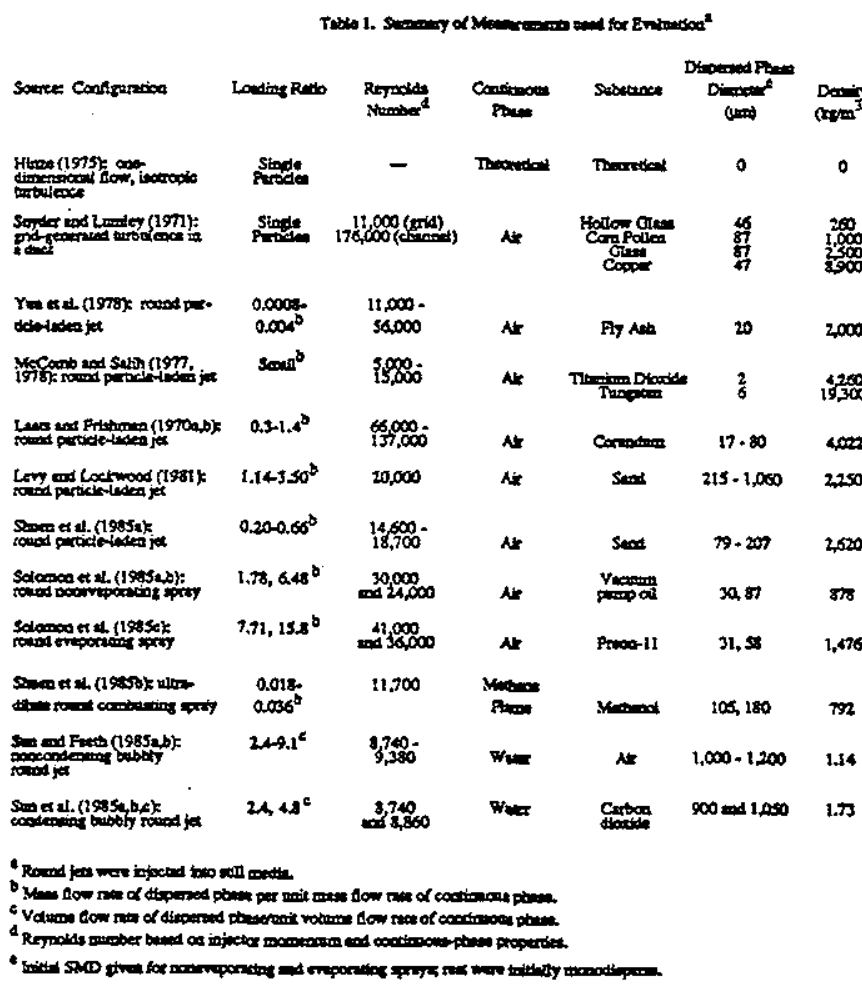

\section{Analysis of Dilute Dispersed Flows}

\section{General Descriotion}

There are three typical methods for analyzing dilute turbulent dispersed flows as follows: (1) locally homogeneous flow (LHF) analysis where interphase transport rates are assumed to be infinitely fast; (2) deterministic separated flow (DSF) analysis where finite interphase transport rates are considered but dispersed phase/turbulence interactions are ignored; and (3) stochastic separated flow (SSF) analysis where both finite interphase transport rates and effects of dispersed phase/turbulence interactions are considered using random-walk computations 
for the motion and transport of the dispersed phase. All three methods will be considered in the following. Some separated-flow analyses employ continuum formulations for the properties of both phases, cf. Williams (1985), however, most use a Eulerian formulation for the continuous phase (which incorporates dispersed-phase source terms) and a agrangian formulation for the dispersed phase. The latter approach will be used here.

In the flows to be considered, the continuous phase is turbulent and must be modeled while averaging over processes on the scale of dispersed-phase elements due to current computational limitations (Faeth, 1983b). A k-e-g turbulence model, in conjunction with the conserved-scalar formalism, is used for this purpose. This tactic can consider a wide variety of processes, including combustion, within a single methodology. This approach originated with workers at Imperial College and their associates (Bilger, 1976,1977; Lockwood and Naguib, 1975). Although improved methods for analyzing turbulence are being sought, evaluation has shown that the present $\mathrm{k}-\varepsilon-\mathrm{g}$ model is reasonably successfui for constant and variable property jetlike flows of interest here (Faeth, 1983b; Jeng and Faeth, 1984a,b; Mao et al., 1980; Shearer et al., 1979). Favre(mass weighted) averages are used in the governing equations since this avoids neglecting terms in the equations for mean properties involving density fluctuations. Interactions between density fluctuations and pressure gradients are ignored in the governing equations for turbulence quantities, however, since considering them vastly increases the complexity and empirism of the formulation and such effects are not very significant for present flows.

Major assumptions for the continuous phase are either typical of current practice or a condition of the experiments, as follows: axisymmetric and steady (in the ean) flow with no swirl; boundary-layer approximations -pply; equal exchange coefficients of all species and heat; buoyancy only affects the mean flow; and negiigible effects of mean kinetic energy and radiation. Flames which are considered are all non-premixed. Under these assumptions the conserved-scalar formalism can be used which implies that instantaneous scalar properties of the continuous phase are only a function of mixture fraction (the fraction of mass at a point which originated from the injector). Expressions relating scalar properties to mixture fraction are called state relationships (Faeth, 1983b).

State relationships can be found by simple adiabatic mixing computations for noncombusting flows, cf., Faeth (1983b) for a number of examples. Early work used similar tactics for flames, computing properties assuming thermodynamic equilibrium. This is effective for fuel-lean conditions but fails for fuel-rich conditions where properties depart from equiblirum due to effects of finite-rate chemistry. Bilger (1977) and Liew et al. $(1981,1984)$, however, have proposed a useful laminar-flamelet approximation which circumvents the difficulty - except near blow out conditions or points of flame attachment. They note that scalar properties in laminar flames (for wide ranges of length scales, residence times and levels of flame stretch) are nearly universal functions of mixture fraction, even through these functions departed from thermodynamic equilibrium estimates for fuel-rich conditions. Thus, correlations found for laminar flames are used for state relationships, viewing scalar properties in turbulent flames

$\checkmark$ the result of a succession of laminar flamelets passing a ven position (or a wrinkled laminar flame fluctuating through the flow). Results using this approach have been encouraging (Liew et al., 1981; Jeng and Faeth, 1984a,b); therefore, the method is used in the following.

\section{LETE Analysis}

LHF analysis will be considered first since the same formulation is used for continuous phase in all three methods. The LHF approximation implies local kinematic and thermodynamic equilibrium, including both phases; therefore, multiphase flows correspond to a variable property single-phase fluid due to variations in concentration of the dispersed phase, even in instances when the properties of each phase are constant. Since this generally involves density variations, properties are presented as Favre averages, defined as follows (Bilger, 1977):

$$
\widetilde{\phi}=\bar{\rho} \phi / \bar{\rho}
$$

where $\phi$ is a generic property and an overbar denotes a conventional time average.

The conserved-scalar formulation, in conjunction

with a $k-\varepsilon-g$ turbulence model, requires solution of governing equations for conservation of mass, momentum and mixture fraction along with modeled governing equations for $k, \varepsilon$ and $g$. These equations can all be written in a common form as follows:

$$
\begin{aligned}
& \mathrm{r} \partial / \partial \mathrm{x}(\tilde{\rho} \tilde{\mathrm{u}} \phi)+\partial / \partial \mathrm{r}(\mathrm{r} \bar{\rho} \tilde{\mathrm{v}} \phi)= \\
& \partial / \partial \mathrm{r}\left(\left(\mathrm{r} \mu_{\mathrm{r}} / \sigma\right) \partial / \partial \mathrm{r}(\phi)\right)+\mathrm{rS} \phi
\end{aligned}
$$

The parameters $\phi$ and $S_{\phi}$ appearing in Eq. (2) are summarized in Table 2 , along with appropriate empirical constants. Reynolds numbers of present flows are relatively large; therefore, laminar transport can be ignored with little error and the turbulent viscosity becomes

$$
\mu_{t}=C_{\mu} \bar{p} k^{2} / \varepsilon
$$

Mean scalar properties are found from the state relationships and the probability density function, $\mathrm{PDF}(\mathrm{f})$, of mixture fraction. In the present case, a clipped-Gaussian Favre-averaged PDF, $\widehat{P}(\mathfrak{f})$, is used. The two parameters needed to specify this distribution are found from the local values of $f$ and $g$ as described by Lockwood and Naguib (1975). Favre-averaged mean quantities are computed, knowing the state relationships, $\phi(f)$, as follows:

$$
\tilde{\phi}=\int_{0}^{1} \phi(f) \widetilde{P}(f) d f
$$

Table 2. Continuows-Phase Source Terms in Govenning Equations

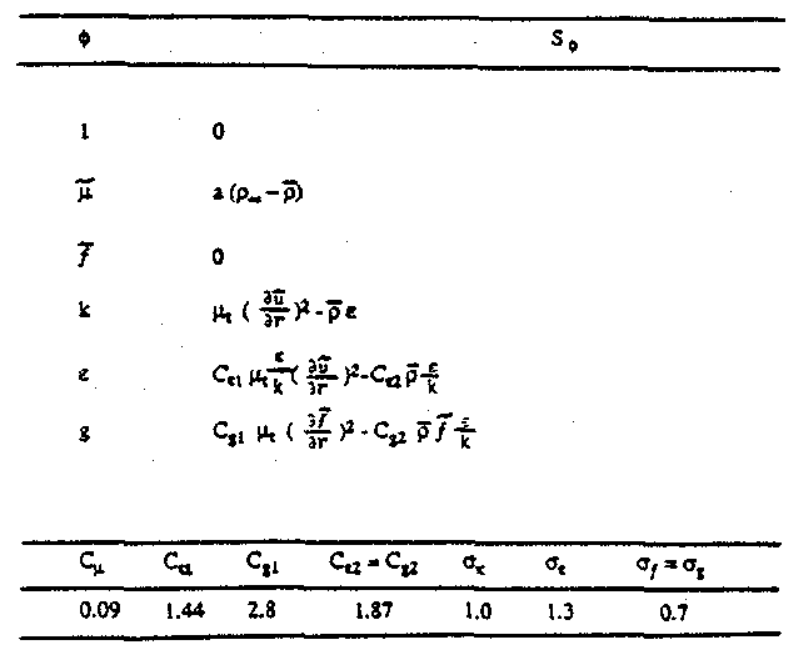


Time-averaged density is needed to solve Eq. (2) and timeaveraged quantities are frequently desired as well. These averages are found from

$$
\tilde{\phi}=\bar{\rho} \int_{0}^{1}(\phi(f) \widehat{P}(f) / \rho(f)) d f
$$

where $\bar{\phi}$ is gotten by setting $\phi=1 \mathrm{in}$ Eq. (5). Original sources should be considered for the details of finding the state reiationships for each flow.

LHFF analysis is not limited to dilute dispersed flows and requires relatively little information concerning initial conditions. Therefore, these computations were initiated at the jet exit in all cases. Faeth (1983b) discusses the prescription of initial conditions and methods used to numerically solve the governing equations.

\section{DSE Analysis}

Both separated-flow models adopt the main features of the LHF model for the continuous phase. The models are limited to dilute dispersed flows; therefore, volume fractions of the continuous phase are near unity and the basic formulation of Eq.(2) and the flow source terms in Table 2 can be retained. The main extension of the continuous-phase analysis for the separated-flow modeis involves the appearance of source terms due to transport from the dispersed phase. Initial conditions are prescribed at the point nearest to the injector where all needed properties can be measured. At this point, the dispersed phase is divided into groups (defined by initial position, size, velocity and direction). Lagrangian computations then track the subsequent life history of each group in the flow field.

Transport from the dispersed phase introduces new source terms, $S_{\mathrm{p} \phi}$, in the governing equations for the continuous phase. These are found by computing property changes for each dispersed-phase group, $i$, as it crosses a computational cell, $j$, and then summing over all groups intersecting a particular cell. Expressions for the $S_{\mathrm{p} \phi}$ are summarized in Table 3. DSF analysis ignores all dispersed phase/turbulence interactions; therefore, the source terms appearing for $k, \varepsilon$ and $g$ in Table 3 are not used with this approach.

While mass and momentum exchange between the phases can be handled directly by the conserved-scalar formalism, there is a conceptral problem with respect to energy transfer. This involves a direct energy loss or gain by the continuous phase, implying that both total enthaipy and mixture fraction are needed to specify instantaneous scalar properties of the continuous phase. For present flows, this is only important for evaporating and combusting sprays, where it was circumvented by using the thin-skin approximation for drop heatup (Faeth, 1977). This implies that the bulk liquid remains at its initial condition while only an infinitely-thin layer at the drop surface is heated (or cooled) during evaporation. This removes the heat loss effect and properties of the continuous phase are once again fully defined by mixture fraction. Sirigano and coworkers have extensively studied energy transport within drops throughout most of their lifetime; therefore, the thin-skin approximation seems more appropriate than the more-widely used uniform drop temperature approximation in any event (Faeth, 1983). In highly-loaded flows, however, this energy transport is important and the conserved scalar formalism must be extended to consider conservation of energy and the correlation between total enthalpy and mixture fraction - a substantial complication.

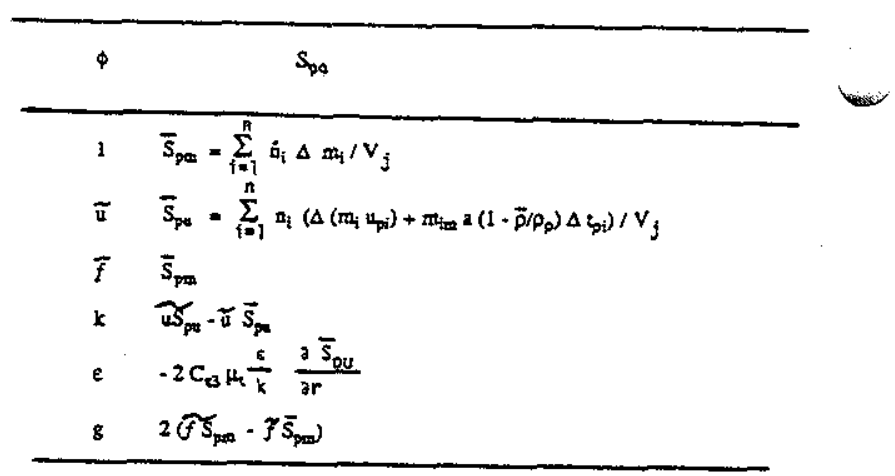

The specific formulation of the equations governing the life histories of dispersed-phase groups will be deferred until the SSF approach is discussed. This aspect of the DSF and SSF analysis is the same except that the DSF computations are based on mean time-averaged, continuousphase properties while the SSF approach seeks to account for instantaneous properties. Time-averaged scalar properties can be found directly from Eq. (5) but the present anaiysis can only provide Favre-averaged velocities. Fortunately, differences between time- and Favre-averaged velocities are relatively small, even in flames (Faeth and Samueison, 1985); therefore, this choice does not introduce significant errors.

\section{SSE Analysis}

There is abundent evidence that both finite interphase transport rates and dispersed-phase/turbulence interactions are important in practical sprays (Faeth, 1983a,b). The LHF method ignores finite interphase transport rates, while the DSF method ignores dispersed phase/turbulence interactions; therefore, neither approach is sufficiently complete. The SSF method was developed in order to circumvent these limitations by extending the DSF method.

There are three main types of dispersedphase/turbulence interactions as follows: (1) turbulent transport or disperson of the dispersed phase; (2) modification of continuous-phase turbulence properties by transport from the dispersed phase, called turbulence modulation by Al Taweel and Landau (1977); and (3) modification of the properties of interphase transport rates by turbulent fluctuations, e.g., the fact that nonlinear interphase transport processes cannot properly be represented using mean propertes in the transport expressions (Faeth, 1983a,b). Initial work on these problems concentrated on turbulent disperson - at the small particle limit (Tchen, 1947; Hjelmfelt and Mochros, 1966; Hinze, 1972, 1975). This implies linear interphase transport, Stokesian flow, and that particles remain within a single fluid element (eddy) during their motion. Later, Elgohbashi and Abou-Arab (1983) extended these ideas to treat turbulence modulation as well. The small particle limit provides a logical approach for developing a better understanding of dispersedphase/turbulence interactions but the assumptions involved are not very realistic for practical sprays. First of all, typical drop Reynolds numbers in sprays are on the order of 100 , well beyond the Stokes regime. Drops have significant velocities relative to the continuous phase as a result; therefore, they do not remain associated with a particular fluid element. The latter effect was recognized by Yudine (1959) and Csanady (1963) and is called the crossing trajectories effect, e.g., the fact that dispersed-phase 
elements and turbulent eddies each follow different trajectories and only interact for a time.

Jurewicz and Stock (1979) propose a more general approach for treating turbulent dispersion using a gradient tiffusion approximation within the Lagrangian formulation or the motion and transport of the dispersed phase. Dukowicz (1980) proposed a related procedure based on a stochastic representation of dispersed-phase diffusion. However, these methods do not provide a means of estimating turbulent diffusivities themselves which is the main problem. Since dispersed-phase diffusivities are influenced by both turbulence and dispersed-phase properties, the accumulation and correlation of appropriate data for these methods is a substantial task, and has not proceeded very far.

Stochastic separated flow methods have been proposed by a number of workers as a way to treat this difficulty, cf., Faeth $(1983 \mathrm{a}, \mathrm{b})$ for a discussion of early work on this problem. The present SSF approach was initially proposed by Gosman and Ioannides (1981) and has been subsequently developed and evaluatedby the author and his associates (Shuen et al., 1983a,b; 1985a,b; Solomon et al., 1985a,b,c; Sun and Faeth, 1985a,b; Sun et al., 1985a,b,c; and Zhang et al., 1985). With this approach, dispersed-phase elements are assumed to interact with a succession of turbulent eddies as they move through the flow. Properties within a particular eddy are assumed to be uniform, but to change in a random fashion from eddy to eddy. Eddy properties are obtained from the continuousphase analysis. The dispersed-phase computations are the same as the DSF approach, except that instantaneous eddy properties are used for the local environment, rather than mean properties. In principle, the method can treat all aspects of dispersed-phase/turbulence interactions but subject to the limitations of the continuous-phase turbulence nodel and the uniform eddy approximation.

The properties of an eddy at the start of interaction between a dispersed-phase element and an eddy are found by making a random selection from the PDFs of velocity and mixture fraction. Velocity fluctuations are assumed to be isotropic, which is implied by $\mathrm{k}-\varepsilon-\mathrm{g}$ analysis, with Gaussian PDF's. The most probable value and variance of these distributions are taken to be the local mean velocities in each direction and $2 k / 3$.. obtained from the continuous-phase solution. The distinction between Favre- and time-averaged velocities was ignored similar to DSF analysis since density/velocity correlations are not provided by the present $\mathrm{k}-\varepsilon-\mathrm{g}$ analysis. This is not a serious problem for mean velocities, as noted earlier, but differences between Favreand time-averages are greater for velocity fluctuations -particularly in flame environments, (Faeth and Samuelson, 1985).

Scalar properties are found by assuming that velocities and mixture fractions are statistically independent for lack of a rational altemative under the approximations of the present $k-\varepsilon-g$ turbulence model. This causes errors in variable density flows where the absolute value of this correlation is on the order of one half in portions of the flow (Faeth and Samueison, 1985). The time-averaged PDF(f), is randomly sampled similar to the velocites, to get an instantaneous value of $f$ for the eddy. Scalar properties of the eddy for this value of $f$ are then obtained from the state relationships.

A dispersed-phase element is assumed to interact with an eddy as long as the displacement of the element with respect to the eddy does not exceed the characteristic eddy size, $L_{e}$, and the time of interaction does not exceed the characteristic eddy lifetime, $t_{e}$. The characteristics eddy parameters are taken to be the dissipation length and time scales which can be obtained from the solution for the continuous phase, as follows

$$
\mathrm{L}_{\mathrm{e}}=\mathrm{C}_{\mu} \mu^{3 / 4} \mathrm{k}^{3 / 2 / \varepsilon}, \mathrm{t}_{\mathrm{e}}=\mathrm{L}_{\varepsilon} /(2 \mathrm{k} / 3)^{1 / 2}
$$

The selection of $\mathrm{L}_{\mathrm{e}}$ and $\mathrm{te}_{\mathrm{e}}$ in Eq. (6) is clearly arbitrary and their values influence turbulent dispersion significantly. Following Gosman and Ioannides (1981), these choices were evaluated by comparing predictions with the fundamental turbulent dispersion results of Hinze (1975) and Snyder and Lumley (1971). Hinze (1975) developed an expression for the diffusion of "marked" fluid particles introduced at a constant rate from a point source in a homogeneous isotropic turbulent flow. Use of Eq. (6) was found to only slightly underestimate the turbulent dispersion of the marked fluid particles found from Hinze's (1975) analysis.

The measurements of Snyder and Lumley (1971) involved dispersion of various types of individual particles which were injected isokinetically into a uniform turbulent flow downstream of a grid. The results of present predictions and these measurements are illustrated in Fig. 1. The SSF predictions are in reasonably good agreement with measurements for both light particles (hollow glass beads), where $t_{e}$ controls the interaction time, and heavy particles (glass and copper beads) where $\mathrm{L}_{\mathrm{e}}$ controls the interaction time.

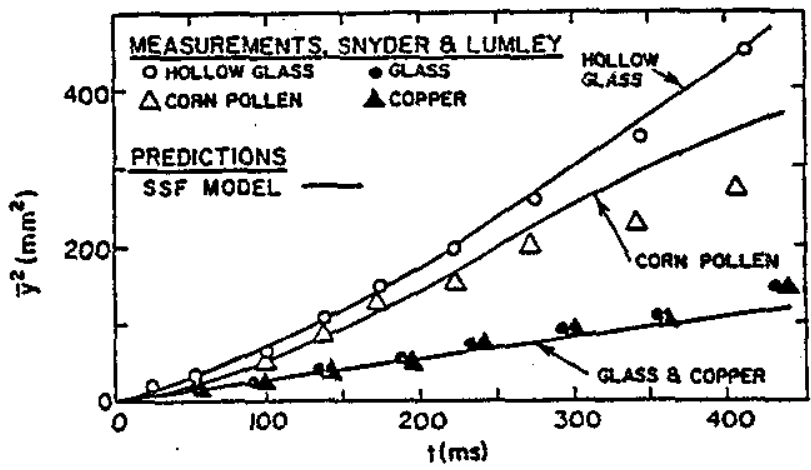

Fig. 1. Particle dispersion in a uniform, grid-generated turbulent flow. From Shuen et al. (1983a).

Assumptions of life-history calculations for the various dispersed-phase groups vary since particle (drop)/gas and bubble/liquid systems are considered in the following; therfore individual sources should be consulted for details. Assumptions common to all are typical of past analysis of dilute sprays (Faeth, 1977, 1983b) as follows: interphase transport is assumed to be quasi-steady for a spherical element, effects of Magus and Saffman-lift forces are ignored; the surface of the dispersed-phase element is assumed to be in thermodynamic equilibrium; empirical expressions are used to treat drag, virtual mass and Basset forces; and interphase energy and mass transfer are treated using stagnant film theory, which allows for finite mass transfer rates, with empirical correction for effects of forced convection.

Under these assumptions, the motion of the dispersed phase can be obtained using the formulation of Odar and Hamilton (1964), later reviewed by Clift et al (1978), as follows: 


$$
\begin{aligned}
& \left(\rho_{p} / \rho+\Delta_{H} / 2\right) d / d t\left(u_{t i}\right)=a\left(1-\rho_{p} / \rho\right) \delta_{1 i}- \\
& 0.75 \mathrm{C}_{\mathrm{D}} / \mathrm{U}_{\mathrm{ri}} / \mathrm{U}_{\mathrm{n}} / \mathrm{d}_{\mathrm{p}}+\Delta_{\mathrm{H}}\left(8 \mathrm{l} \mathrm{v} / \pi \mathrm{d}_{\mathrm{p}}{ }^{2}\right)^{1 / 2} \\
& \mathrm{t} \\
& \int(t-\xi)^{-1 / 2} d / d \xi\left(u_{r i}\right) d \xi \\
& t_{0}
\end{aligned}
$$

where $\delta_{\mathrm{ji}}$ is the Kroneker delta function and $\mathrm{i}=1$ represents the free fall (or rise) direction of the dispersed phase. The two-terms in the LHS of Eq. (7) represent accelerations due to particle and virtual mass. Only the fomer is important for particle (drop)/gas flows; only the latter for bubble/liquid flows. The terms on the RHS of Eq. (7) represent buoyancy, drag and Basset history forces. The Basset history force is important for bubble/liquid flows but can be ignored for particle (drop)/gas flows with little error. The parameters $\Delta_{A}$ and $\Delta_{H}$ were empirically correlated by Odar and Hamilton (1964) as a function of the accleration modulus

$$
M_{A}=d / d t\left(u_{t}\right) d_{p} / u_{T}^{2}
$$

The values of $\Delta_{\mathrm{A}}$ and $\Delta_{\mathrm{H}}$ vary between 1.0-2.1 and 1.000.48 --the former values being the correct limit for the classical Basset - Boussinesg-Osceen (B-B-O) formulation of Eq. (7). Drag coefficients were obtained either from the values for solid spheres (particles/drops) summarized in Faeth (1977,19836) or for bubbles (Moore, 1965; Clift et al. 1978). The position of each dispersed-phase group is found by integrating the group velocity as a function of time.

The formulation of interphase transport of mass and energy is too variable to show all cases here; therefore, the following will be limited to drop vaporization to illustrate the general approach. In addition to the previous assumptions, the gas-phase Lewis number is assumed to be unity; only concentration diffusion is considered with equal binary diffusivities; and the Chapman gas property approximations are used, e.g., ideal gas mixture with $\rho^{2} D, \rho \lambda$ and $C_{p}$ constant. Then the rate of change of mass of a drop in group $i$ is given by

$$
\begin{gathered}
d / d t\left(m_{p i}\right)=-2 \pi(p D)_{\text {refd }} d_{p} N \\
\ln \left(\left(Y_{F s}-Y_{F_{\infty}}\right) /\left(1-Y_{F S}\right)\right)
\end{gathered}
$$

where $Y_{F s}$ is the mass fraction of drop vapor at the liquid surface and $\mathrm{N}$ is the correction factor allowing for drop motion with respect to the gas phase (forced convection correction), as follows (Faeth, 1983b)

$$
\mathrm{N}-1=0.276 \operatorname{Re}^{1 / 2} \mathrm{Pr}^{1 / 3} /\left(1+1.232 /\left(\operatorname{Re} \operatorname{Pr}^{4 / 3}\right)\right)^{1 / 2}
$$

Under the thin-skin approximation all the heat reaching the liquid surface is used to preheat the liquid to the surface temperature and vaporize it, e.g., no bulk heating is considered. This yields the following relationship between surface temperature and mass fraction

$$
Y_{F_{S}}=\left(C_{p}\left(T_{\infty}-T_{S}\right)+Y_{F_{\infty}} L_{S}\right) /\left(C_{p}\left(T_{\infty}-T_{S}\right)+L_{S}\right)
$$

where $I_{r}$ is the total enthalpy rise of vaporization

$$
L_{s}=C_{P f}\left(T_{s}-T_{0}\right)+h_{f g s}
$$

A second expression relating the surface vapor mass fraction and temperature is provided by the vapor pressure characteristics of the liquid

$$
\mathrm{Y}_{\mathrm{Fs}}=\mathrm{f}\left(\mathrm{T}_{\mathrm{S}} \text {, pressure }\right)
$$

Simultaneous solution of Eqs. (11) and (13) then yields $T_{S}$ and $Y_{F s}$ for any imposed ambient conditions. Since oulk heating has been ignored, drop diameter and mass are related as follows

$$
\left.\mathrm{d}_{\mathrm{pi}}=\left(6 \mathrm{~m}_{\mathrm{pi}} / \pi \rho_{\mathrm{fo}}\right)\right)^{i / 3}
$$

Life-history computations are strongly influenced by reference conditions used to determine transport properties and the specific correlations of the properties themselves. During work reported here, properties were selected by matching predicted and measured life histories for single dispersed elements (particles, drops or bubbles) at test conditions representative of the multiphase flow. This involves selecting an optimum weighting parameter, $\beta$, to define the property reference state

$$
\phi_{\text {ref }}=\beta \phi_{S}+(1-\beta) \phi_{\infty}
$$

where $\phi$ is a generic factor representing either species mass fraction or temperature.

Equations (7)-(15) were solved numerically using a second-order Runge-Kutta algorithm. Details of these computations are presented elsewhere (Shuen, 1983a). Closure with respect to the number of dispersed-phase groups needed for statistically-significant results varies with the flow. Typical values are ca 1000 groups for the DSF approach and ca 5000 groups for the SSF approach. Solution for the properties for all groups immediately yields the dispersed-phase source terms needed for the continuous phase -- summarized in Table 3. Aside from the preseciptions for $\mathrm{L}_{\mathrm{e}}$ and $\mathrm{t}_{\mathrm{e}}$, which have already been discussed the only new empirism involves $C_{\varepsilon 3}$ which appears in the dispersed-phase source term in the $\varepsilon$ equation (this term was found making other approximation typical of models of this equation (Shuen et al, 1985a)).

\section{STRUCTURE OF DILUTE DISPERSED FLOWS}

In the following, we shail consider the performance of LHF, DSF and SSF analysis. A variety of dilute dispersed flows will be considered, as follows: particleladen jets, nonevaporating sprays, evaporating sprays, combusting sprays and bubbly noncondensing and condensing jets. The test conditions for the flows are summarized in Table 1. The test flows are dilute and effects of turbulence modulation are small; therefore, SSF computations in the following ignore turbulence modulation unless noted otherwise. This implies that the particle source terms in the goveming equations for $\mathrm{k}, \varepsilon$, $\mathrm{g}$, listed in Table 3 , are ignored.

\section{Particle-Laden Jets}

The significance of turbulent dispersion in particleladen jets can be seen from results appearing in Fig. 2 . The particle concentration measurements of Yuu et al (1978) are illustrated along with predictions using all three models. Only the range of streamwise positions where data were measured was reported; therefore, predictions are illustrated 
for the limits of this range. Particle properties at the jet exit were estimated from the nozzle geometry since they were not reported. The estimated initial velocities appear on the figure.

The rate of particle spread is overestimated using the HF analysis, since effects of relative velocities between the of hases (slip) are ignored. Neglecting slip causes the particle response to turbulent fluctuations, the mechanism of turbulent dispersion, to be overestimated. This also reduces streamwise particle velocities in the flow field, and the increased residence time causes further overestimation of particle spread rates.

The DSF analysis underestimates particle spread rates in Fig. 2. In this case, particle spread is only caused by initial particle radial velocities and by drag in the radial direction due to the mean radial velocity of the gas phase. Both of these velocities are small in comparison to gas-phase radial fluctuating velocities, which are responsible for turbulent dispersion. Furthermore, since the radial velocities of particles eventually are dominated by gas-phase radial velocities, particles tend to accumulate in regions were $\mathrm{v}=0$, e.g., their behavior is similar to particles in laminar flow, c.f., Soo (1967). In jets, $v=0$ at the centerline (unstably) and roughly half-way between the axis and the flow edge. It takes time for this process to develop, and effects of gravity modify the effect. Thus, the trend can only be seen indirectly in Fig. 2, by a tendency for the profiles of particle concentration to become progressively narrower (in terms of

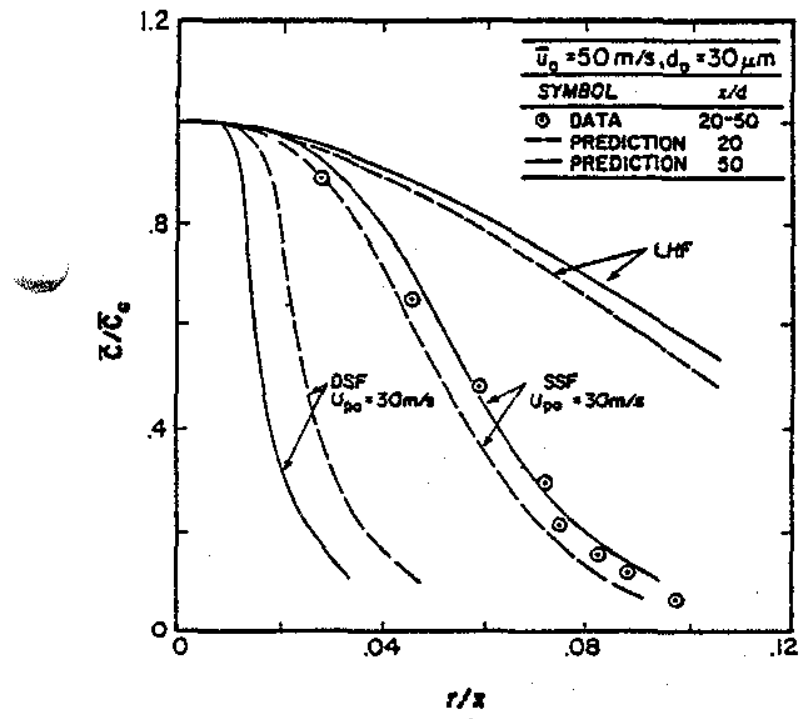

Fig. .2 Particle dispersion in a round turbulent jet From Shuen et al. (1983b).

$r / x$ ) with increasing streamwise distance, rather than the opposite trend given by the LFF and SSF analyses.

In contrast to the LHF and DSF methods, SSF predictions are in reasonably good agreement with the measurements illustrated in Fig. 2. This suggests that both finite interphase transport rates and turbulent dispersion were important for this flow. Evaluation of the SSF method with these data, however, is not very definitive due to uncertainties in initial particle velocities.

Shuen et al (1983b) extended evaluation of the ralyses using measurements reported by $\mathrm{McComb}$ and talih $(1977,1978)$, Lats and Frishman $(1970 \mathrm{a}, \mathrm{b})$ and Levy and Lockwood (1981). Findings were similar to Shuen et al (1983a). LHF and DSF analyses over- and under-estimated particle spread rates while the SSF approach yielded encouraging predictions. Persistent uncertainties in initial conditions, however, prevented definitive evlaluation of the SSF method. Measurements by Modarress et al (1984) were more complete, but attempts to evaluate the analyses with these data were inhibited since small streamwise pressure gradients significantly influenced these flows but were not reported (Zhang et al., 1985).

Many of the experimental difficulties were resolved in the particle-laden jet study reported by Shuen et al (1985a). Particles in these flows were nearly monodisperse. Mean and fluctuating phase velocities were measured in free particle-laden jets using laser Doppler anemometry. Distributions of particle mass fluxes were measured by isokinetic sampling at the mean streamwise gas velocity.

Measured and predicted (LHF and SSF methods) mean particle velocities along the axis of the particle-laden jet studied by Shuen et al (1985a) are illustrated in Fig. 3. Mean gas velocities for these conditions roughly corresponded to the LHF predictions illustrated in Fig. 3. Thus, the significant effect of slip can only be adequately treated using the separated flow models. Results illustrated in Fig. 3 are typical of many multiphase jets (Faeth, 1983b). The continuous-phase rapidly decelerates beyond the end of the potential core (or near the jet exit if no core is present), at a rate which can only be followed by smail particles -. typically less than $10 \mu \mathrm{m}$ in diameter for particle (drop)/gas flows. Few practical sprays are atomized to this level; therefore, LHF analysis is generally not very effective for quantitative predictions in dilute sprays.

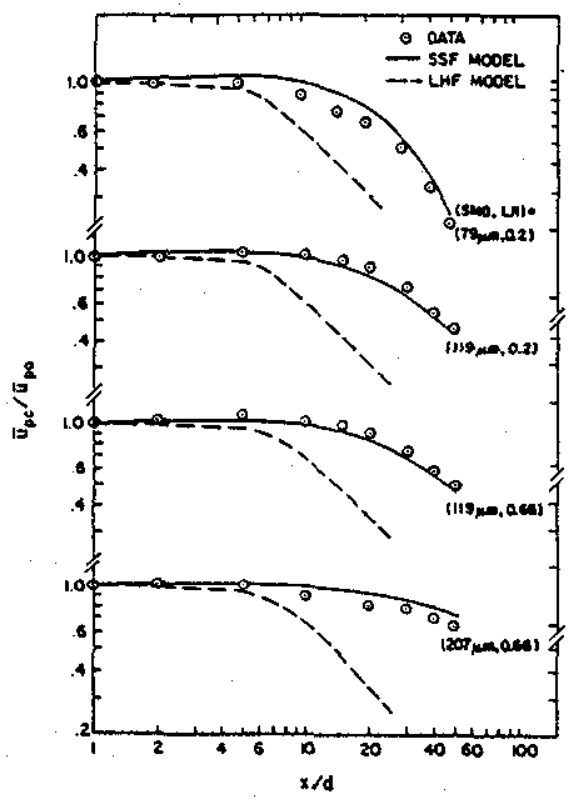

Fig. 3. Mean particle velocities along the axis of it round turbulent jet. From Shuen et al. (1985a).

Typical radial profiles of mean and fluctuating particle properties, from Shuen et al (1985a), are illustrated in Fig. 4. Predictions of the LHF and SSF analyses are illustrated along with the measurements. DSF predictions of mean properties (the only particle properties this model provides) are also shown. Trends of mean properties are similar to the resuits discussed on connection with Fig. 2. Predictably, the LHF model overestimates particle velocity fluctuations due to neglect of slip. The SSF predictions of particle velocity fluctuations are reasonably good, which is consistent with its representation of turbulent particle dispersion. 


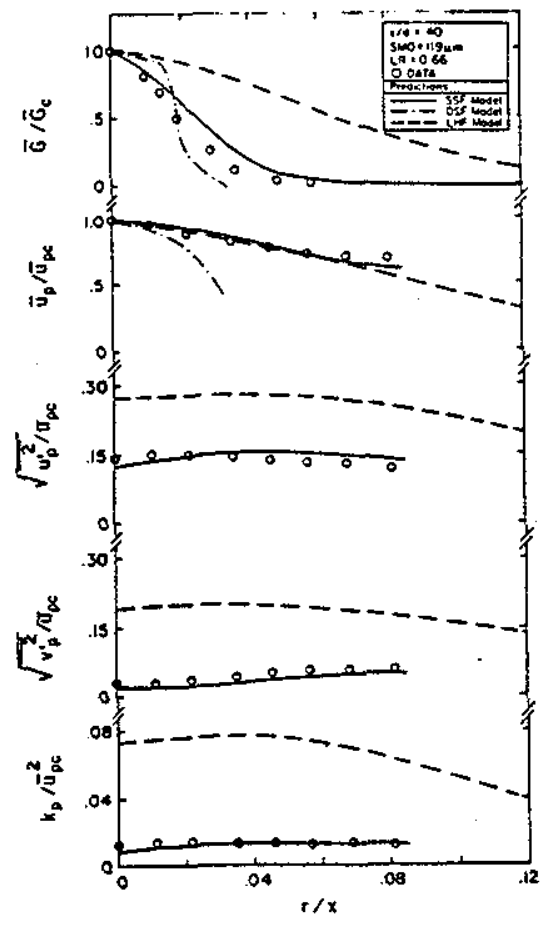

Fig. 4. Radial variation of mean and fluctuating particle properties in a round turbulent jet. From Shuen et al. (1985a).

An interesting effect seen in Fig. 4 is that particle velocity fluctuations are anisotropic, with streamwise fluctuations being much larger than radial fluctuations, even though the analysis used to predict particle fluctuations assumes isotropic velocity fluctuations for the continuous phase. This is caused by radial transport of particles from regions having different mean streamwise particle velocities, followed by relatively slow relaxation to the new state via the indirect mechanism of drag from the continuous phase. This mechanism is somewhat similar to the phenomena causing anisotropic velocity fluctuations in single-phase turbulent jets. For the conditions of Fig. 4, the SSF approach gives nearly quantitative predictions of levels of anisotropy of particie velocity fluctuations, however, we shall see that this is not always the case.

\section{Nonevaporating Sprays}

Consideration of sprays vastly complicates both measurements and predictions, since drop properties must be segregated by size. Results from Solomon et al. (1985a,b) for nonevaporating sprays will be considered since they are reasonably complete. Measurements involved air atomization of vacuum pump oil (insuring negligible evaporation) to yield a free spray. Mean and fluctuating gas phase velocities were measured with an LDA, while drop size and velocity distributions were measured using multiflash photography. Mean drop mass flux was measured by isokinetic sampling at the mean streamwise gas velocity. The dense-spray region near the injector was not considered, due to lack of adequate spatial resolution, opacity to optical diagnostics, and the presence of irregularly-shaped liquid elements (ligaments, etc.); therefore, both measurements and predictions were confined to $x / d \geq 50$. The position $x / d=50$ was used to find initial conditions for the separated flow analyses. LHF calculations were started at the injector exit, however, since this approach is not fundamentally limited to dilute sprays.
Some of the complexities of polydisperse sprays are illustrated in Fig. 5. Predicted (DSF and SSF methods) and measured mean streamwise drop velocities along the axis are plotted as a function of drop size and $x / d$. Drop velocities decrease with both size and distance from the injector. At $x / d=50$ and 100 , drops with $d_{p}<30 \mu \mathrm{m}$ had velocities up

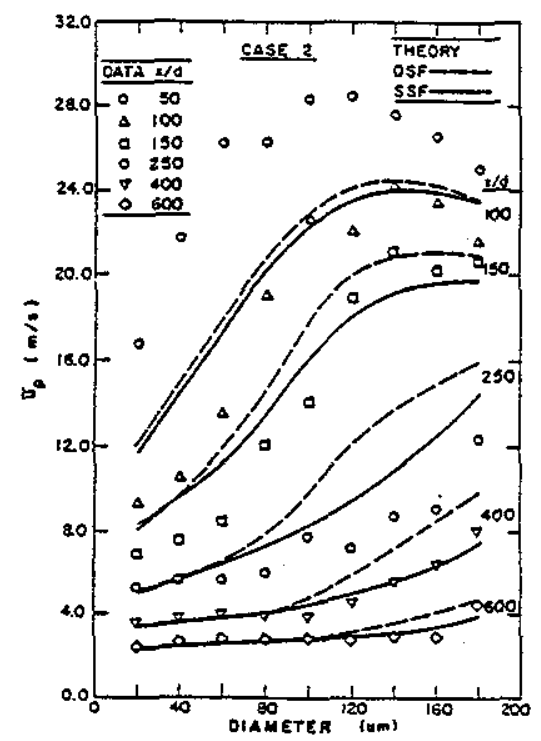

Fig. 5. Mean streamwise drop velocities in a round non- evaporating spray. From Solomon et al. (1985b).

to $30 \%$ less than the gas velocity while the largest drops had velocities up to twice the gas phase value. Far downstream, however, at $x / d=600$, velocity differences become small -approximating LHF flow. SSF predictions in Fig. 5 yield a more rapid deceleration than DSF predictions for each drop size. This is due to the nonlinearity of the drag law interacting with turbulent fluctuations -- one of the dispesedphase/turbulence interactions discussed earlier. The SSF method yields better predictions than the DSF method, since it uses instantaneous properties and only averages over particle groups. ORourke and Bracco (1980) propose an alternative for use with DSF analysis, however, this approach has not been evaluated as yet.

Predictions (all three models) and measurements of mean liquid flux for the nonevaporating sprays are illustrated in Fig. 6. An interesting property of these results is the extraordinary width of the flow just downstream of the dense-spray region. A single-phase flow, which should spread more rapidly than the dispersed-phase distribution, would roughly have a concentration profile similar to the LHF predictions in Fig. 6 . The spray is roughly twice as wide near $\mathrm{x} / \mathrm{d}=50$ and only approaches the LHF profile in the far field. The fact that the unusual width is associated with the near-injector region suggests a dense-spray mechanism, although some unmonitored instability of the injector passage cannot be ruled out. Drop collisions and drop breakup, which are frequent in polydisperse dense sprays, could be responsible since this provides a means of efficiently converting streamwise to radial drop momentum. The phenomenon deserves further study since it strongly influences the initial conditions of the dilute portion of the spray.

The unusual width of the dense spray region cannot be sustained as the spray becomes dilute and the liquid mass flux distributions evolve toward LHF predictions as $x / d$ increases for the results pictured in Fig. 6. Effects of turbulent dispersion are significant during this evolution; 


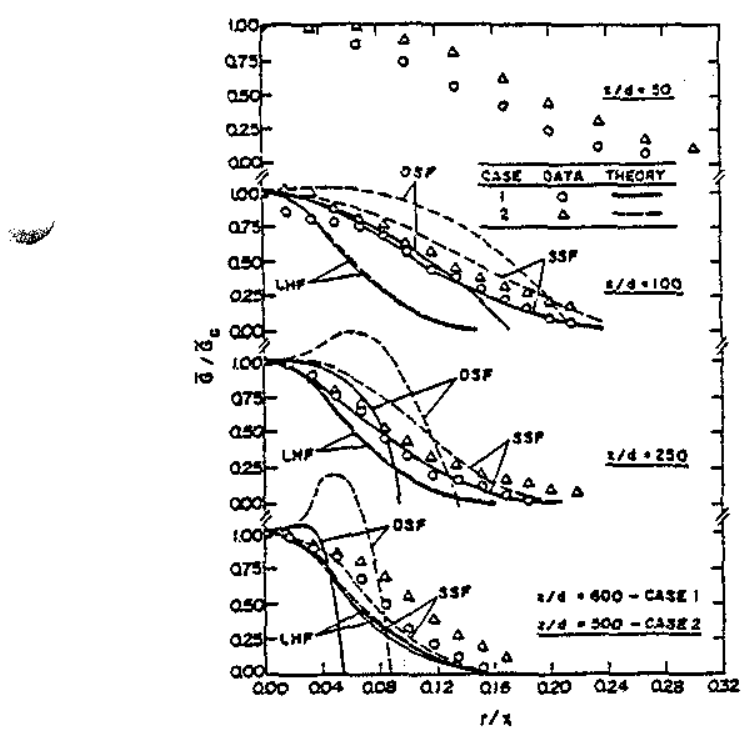

Fig. 6. Mean liquid flux distributions in a round nonevaporating spray. From Solomon et al. (1985a).

therefore, DSF predictions show a more rapid narrowing of the flow than SSF predictions, which are in better agreement with measurements. DSF predictions also yield pathological concentrations of drops at radial distances were $v=0$, e.g. for $\mathrm{s} / \mathrm{x}=0.06-0.10$ at $\mathrm{x} / \mathrm{d}=600$. This behavior was discussed earlier. SSF predictions of mean liquid fluxes are also poorer than for other parameters of these flows. Computations showed that this was due to the very high sensitivity of liquid fluxes to uncertainties of initial conditions (Solomon et al., 1985a,b).

Turbulent dispesion and relative velocities vary with drop size; therefore, the size distribution of drops changes as the flow develops in polydisperse sprays. This is illustrated in Fig. 7, where the Sauter Mean Diameter (SMD) along the

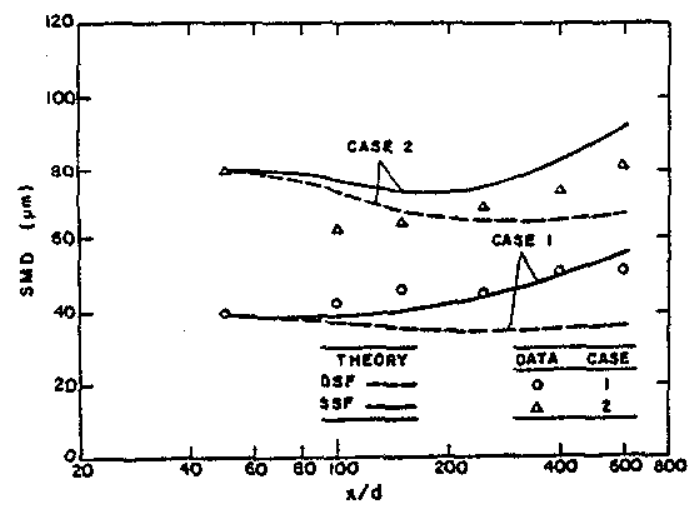

Fig. 7. SMD along the axis of round nonevaporating sprays. From Solomon et al. (1985b).

axis of the nonevaporating sprays is plotted. The fact that large drops pass through the flow more rapidly and are less influenced by turbulent dispersion than small drops causes a progressive increase of SMD along the axis. The SSF analysis appears to represent this effect reasonably well.

Effects of turbulence modulation can be seen in the predictions and measurements of gas phase velocity fluctuations which are plotted in Fig. 8 . The present continuous-phase analysis only provides $\mathrm{k}$; therefore, velocity fluctuation predictions were obtained assuming the usual levels of anisotropy found in single-phase jets, e.g., $u^{\prime 2}: v^{\prime 2}: w^{\prime 2}=k: k / 2: k / 2$ (Wygnanski and Fiedler, 1969). Recall that $x / d=50$ is the initial condition for predictions, where $k$ is matched to the measurements. It is evident that measured levels of anisotropy are much larger than the usual levels seen in jets at this position near the dense-spray region. Farther into the dilute spray region, however, effects of drops on turbulence properties decrease and anisotropy levels approach those of single-phase jets.

Another effect, attributable to turbulence modulation, is the relativeiy low levels of turbulent fluctuations near the dense spray region seen in Fig. 8. This is particularly noticable near the axis of the flow. One reason for this behavior is the relatively broad mean velocity profiles, which reduces turbulent production by shear forces, near the dense spray region. The predictions model this effect but still overestimate turbulence levels near the dense spray region. Calculations considering turbulence modulation provide better results. The empirical constant $C_{e 3}$, needed in the terms representing turbulence modulation in Table 3, is not known very well. Available evidence, however, suggests that its value is small, ca. $0.01-0.1$ (Sun et al., $1985 \mathrm{a}, \mathrm{b}, \mathrm{c}$ ). Including the turbulence modulation terms in the SSF analysis and using this constant yields reductions in $k_{c}$, and thus the components of velocity fluctuations, and improves the comparison between predictions and measurements (Solomon et al., 1985b). The quantitative effect of turbulence modulation in the dilute spray region, however, was comparable to uncertainties in predictions due to uncertainties in initial conditions. Thus, the measurements of Solomon et al $(1985 \mathrm{a}, \mathrm{b}, \mathrm{c})$ are not adequate to definitively study turbulence modulation phenomena.

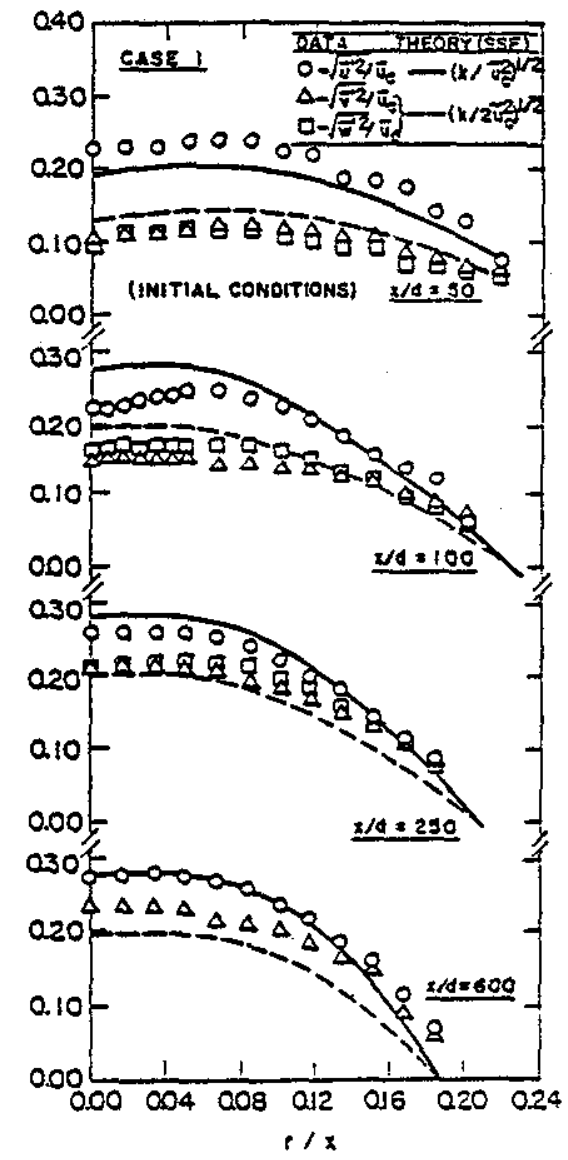

Fig. 8. Gas-phase velocity fluctuations in a round nonevaporating spray. From Solomon et al. (1985b). 
Solomon et al. (1985c) extended their work to evaporating sprays, using the same apparatus and test methods as their study of nonevaporating sprays. Evaporation influences scalar properties of the gas phase; therefore, even the separated flow models must employ the conserved scalar formulation in this case. These experiments involved injection of liquid Freon-11 into still air, using an air atomizing injector.

Predicted and measured profiles of total (gas and liquid) mean Freon-11 concentrations are illustrated in Fig. 9. Similar to the nonevaporating sprays, the flow is unusually wide near the dense spray region. The behavior is somewhat less pronounced, however, than for nonevaporating sprays since drops rapidly evaporate as they reach the edge of the flow - limiting their penetration into the surroundings.

DSF predictions in Fig. 9 exhibit peaked profiles, due to neglect of turbulent dispersion of drops. LHF and SSF predictions are in better agreement with the measurements. LHF analysis yields better results in this case since Freon-11 vapor tends to dominate the total concentration measurements for present test conditions.

Predicted (DSF and SSF analysis) and measured SMD along the axis for the sprays are illustrated in Fig. 10. For nonevaporating sprays, SMD increased with increasing distance from the injector, c.f. Fig. 7. For evaporating sprays, however, this is counteracted by drop evaporation. Thus, the SMD remains relatively constant along the axis until the last stages of drop vaporization for these test conditions - trends which are represented reasonably well by both theories.

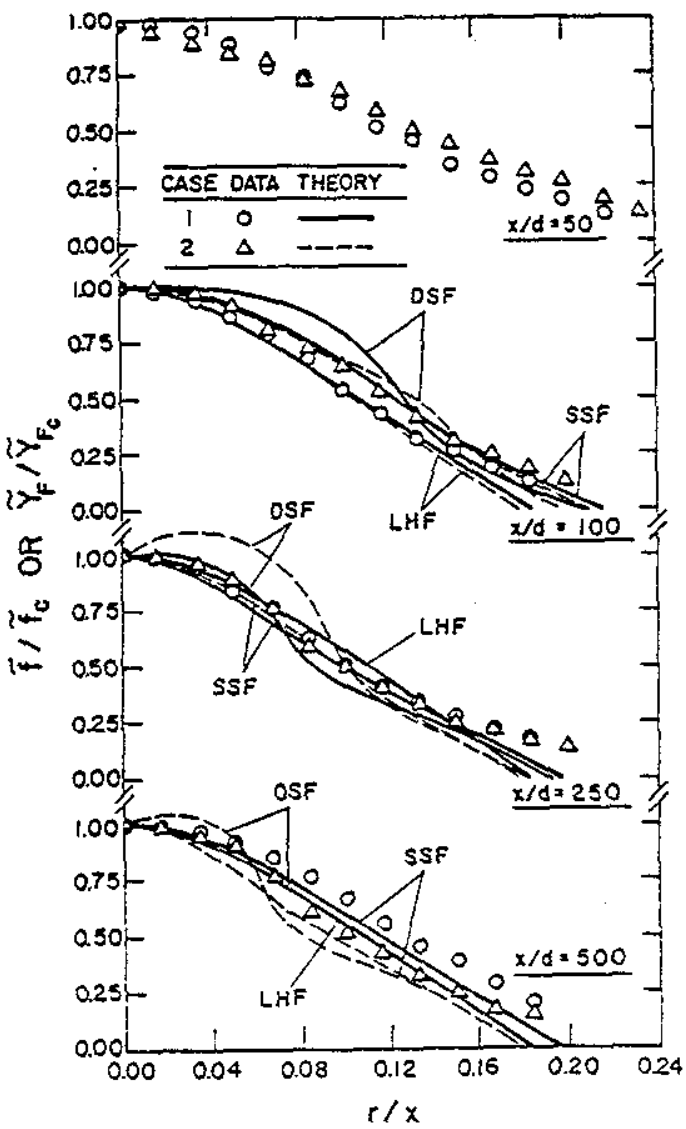

Fig. 9. Distributions of total Freon-11 concentrations in round evaporating sprays. From Solomon et al. (1985c).

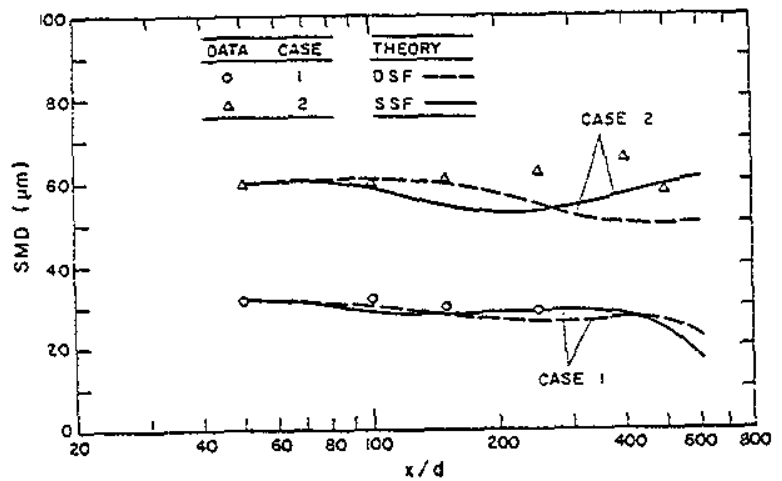

Fig. 10. SMD along the axis of round evaporating sprays. From Solomon et al. (1985c).

Predictions and measurements of gas-phase turbulent kinetic energy are illustrated in Fig. 11. Effects of turbulence modulation are evident for both sprays near $x / d=$ 50. Spray 1 is more lightly loaded, this results in smaller reductions of $\mathrm{k}$ levels near the dense spray region and more rapid disappearance in turbulence modulation effects with increasing distance from the injector. These measurements only employed amplitude discrimination of the LDA to eliminate effects of drops on measurements of gas phase properties; therefore, uncertainties are introduced due to grazing collisions of large drops with the measuring volume. Modarress et al. (1984) describe an improved phase discrimination system for continuous-phase LDA measurements; systems like this are recommended for quantitative work in the future. Nevertheless, large drops had very high slip velocities in the test sprays and it is difficult to see how extraneous signals from them could cause measurements of reduced turbulence levels. Thus the lower levels of $k$ near the dense spray regions of the nonevaporating and evaporating sprays provide reasonably good evidence of effects of turbulence modulation.

\section{Combusting Sprays}

The combusting spray experiments of Shuen et al. (1985b) involved ultra-dilute conditions throughout the flow. Initially monodisperse methanol drops were injected vertically upward at the base of a methane-fueled diffusion flame burning in still air. The methane flame had been extensively studied by Jeng and coworkers (1982, 1984a), establishing predictive methods for the flow using the conserved-scalar formalism in conjunction with the laminarflamelet approximation. The methanol drops only perturbed this flow; therefore, their environment was well known throughout the flame. Mean and fluctuating drop velocities were measured using LDA; drop sizes were measured using flash photography; and drop number fluxes were measured using Mie scattering. Drop histories to any point in the flow vary due to effects of turbulence; therefore, drop sizes are not monodisperse at any point other than the exit. This was not considered in the measurements; drop properties were simply averaged over all sizes at each point. Computations were averaged in the same manner so that predictions and measurements could be compared.

Only separated flow predictions will be reported in the following. For this ultra-dilute flow, drop properties are controlled entirely by interphase transport rather than mixing of the flow as a whole; therefore, the LHF method indicates 


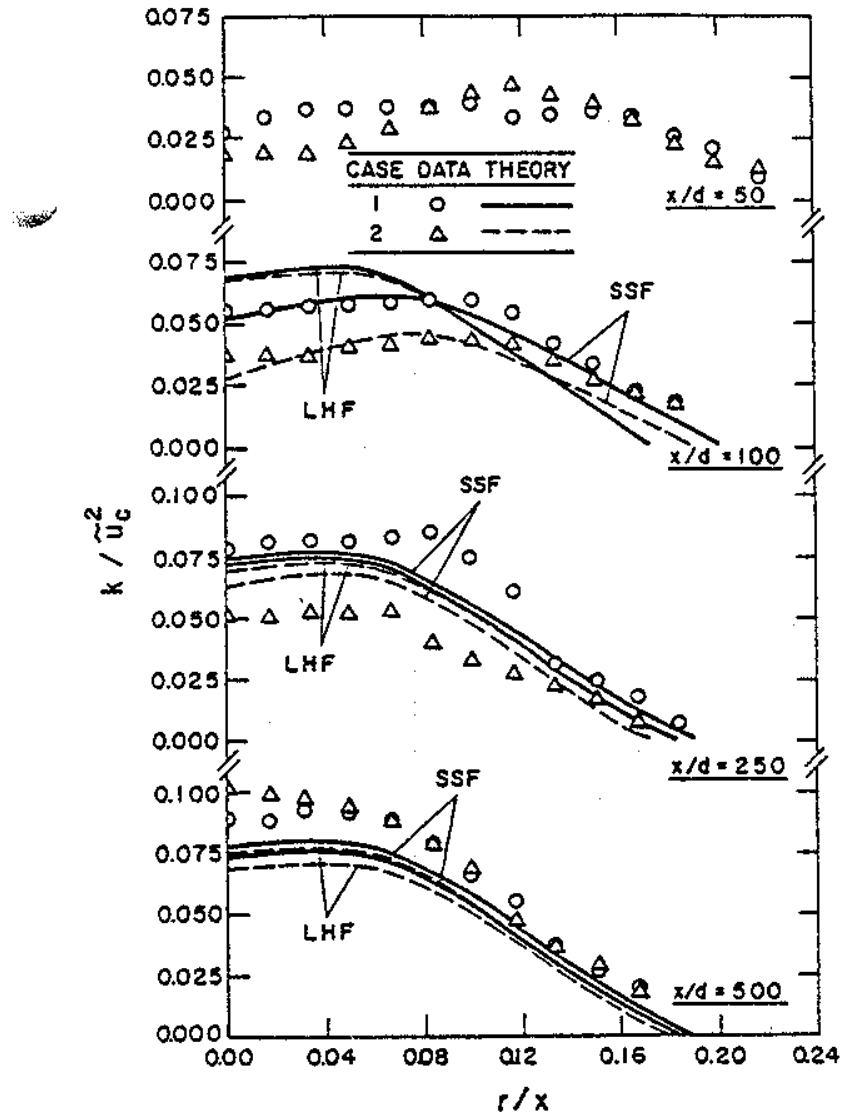

Fig. 11. Distributions of gas-phase turbulence kinetic energy in round evaporating sprays. From Solomon et al. (1985c).

that the drops evaporate completely very near the injector exit which is clearly erroneous.

The possible existence of envelope flames around each drop is a controversial matter for analysis of combusting sprays (Faeth, 1983b). In fuel-rich regions this is clearly not possible, but could occur when drops interact with fuel-lean eddies. Szekely and Faeth (1983) studied drops supported at various positions in a turbulent diffusion flame to provide some information concerning the issue. They found that differences in transport rates between evaporating and combusting drops were relatively small (less than $10-20 \%$ ) until the mean fuel-equivalence ratio of the flame environment dropped below $90 \%$.

Drops in the tests of Shuen et al. (1985b) penetrated beyond this condition, but the following predictions still neglect the effect of envelope flames. Additional calculations considering envelope flames to be present, for fuelequivalence ratios less than unity, did not indicate significant effects of envelope flames. However, this may not always be the case. Predictions considering envelope flames are problematical since there is very little information available concerning their ignition and extinction properties in flame environments.

Measurements of mean gas-phase (time averaged) and drop (particle-averaged) velocities along the axis are plotted in Fig. 12. Drop velocities for both sprays tested are shown along with SSF predictions. Predicted gas velocities are Favre-averages, however, differences between time- and
Favre-averaged mean velocities are not very large, as noted earlier. Gas velocities are greater than drop velocities at the burner exit, but decrease rapidly due to mixing with the surroundings. Near the injector, drops have significant inertia and their velocities only increase gradually due to drag from the gas. Near the tip of the flame ( $\mathrm{x} / \mathrm{d} \mathrm{ca} .120$ ), however, drops become small and they rapidly approach gas velocities. The SSF analysis does a reasonably good job of predicting these trends.

Predicted (DSF and SSF methods) and measured mean drop number fluxes (both time averages) are illustrated in Fig. 13. The initially larger drops have wider profiles even through they are less responsive to turbulent dispersion. This occurs since they are able to penetrate farther into the flame zone before evaporating. SSF predictions provide the same ordering of spread rates and are in fair agreement with the measurements. The DSF

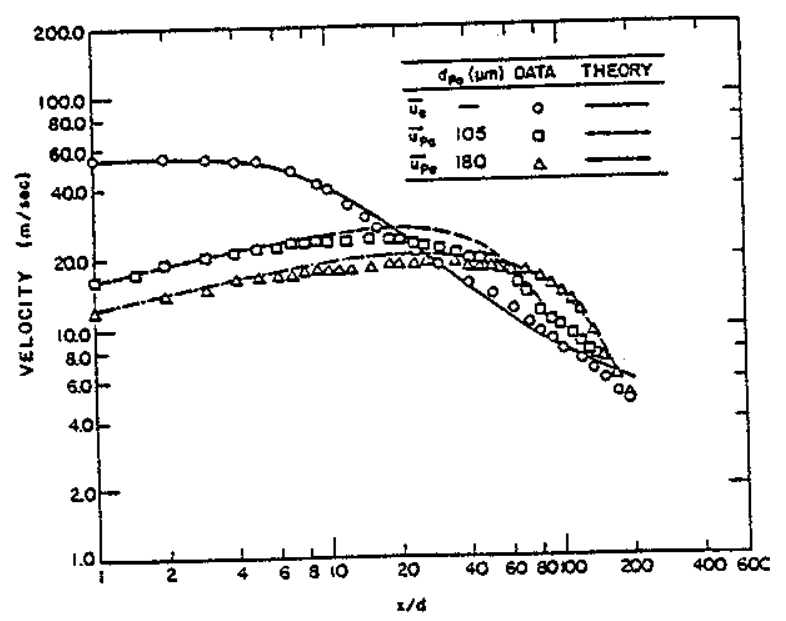

Fig. 12. Mean phase velocities along the axis of round, ultra-dilute, combusting sprays. From Shuen et al. (1985b).

predictions yield incorrect ordering of the spread rates and are not very effective, similar to the flows considered earlier.

Measured (time- and particle averages) and predicted (SSF method) phase velocity fluctuations along the axis are illustrated in Fig. 14. As before, gas velocity fluctuations were computed by using the normal levels of anisotropy found in turbulent jets. Predicted gas phase velocity fluctuations are Favre-averages while the measurements are time averages. However, this distinction is comparable to experimental uncertainties, as are the differences between predictions and measurements.

Particle velocity fluctuations plotted in Fig. 14 show very high leveis of anisotropy, much larger than predicted. Radial particle velocity fluctuations are predicted reasonably well, which is consistent with the satisfactory predictions of turbulent dispersion. Streamwise drop velocity fluctuations are substantially underestimated, however, probably due to the assumption of isotropic turbulence when eddy properties are selected for SSF analysis. Near the bumer exit, drop velocity fluctuations are small in comparison to the gas phase, due to drop inertia. At the end of drop lifetime $(x / d$ ca. 90-120), however, the remaining small drops can respond rapidly and approach flame properties. 


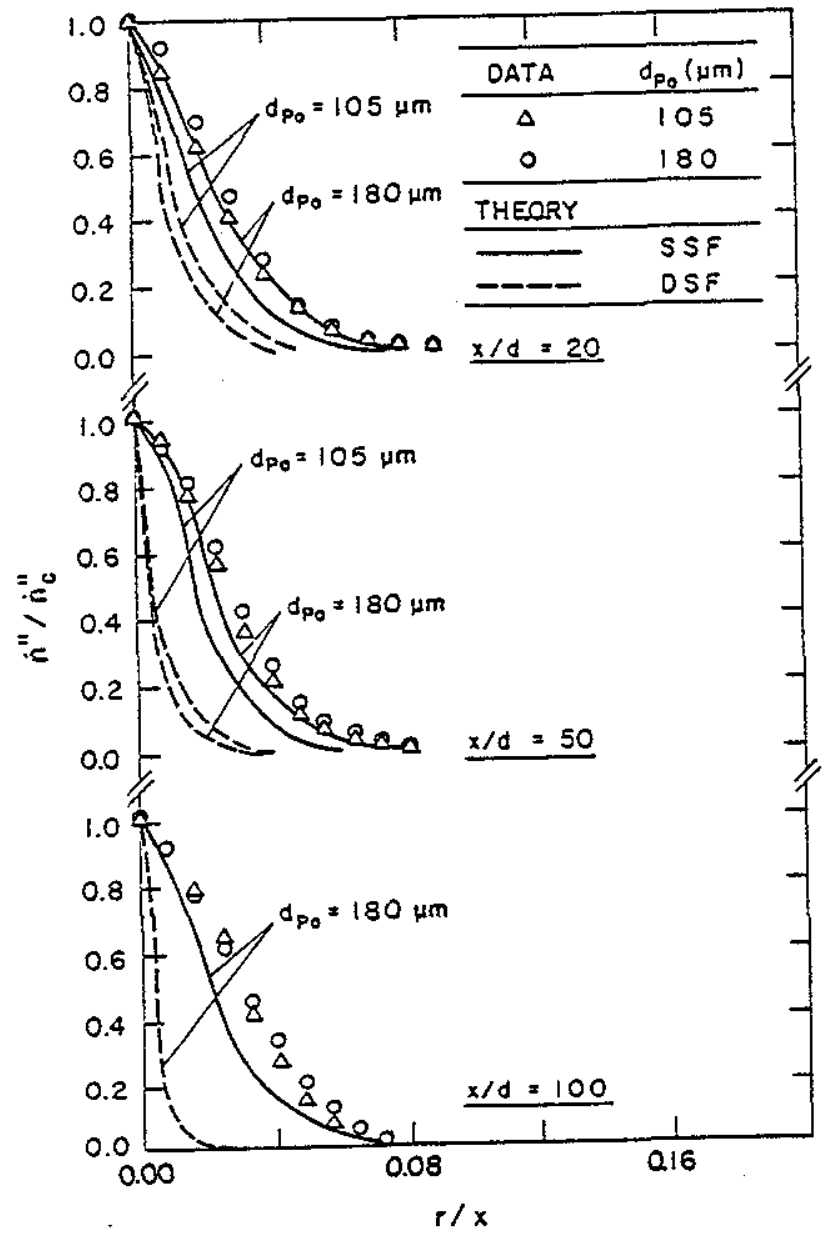

Fig. 13 Drop number flux distributions in round, ultra-dilute combusting sprays. From Shuen et al. (1985b).

Bubbly Jets

Present methods of analysis were largely developed for particle-laden jets and sprays; therefore, bubbly jets provide a challenging test of the approach. In this case, bubble inertia is negligible while virtual mass and Basset history forces become important. Thus, considering such flows provides an indication of the robustness of the analysis as weil as insights gained by studying multiphase turbulent jets from a different perspective.

Predictions and measurements for condensing bubbly jets by Sun et al. $(1985 a, b, c)$ will be considered. An earlier study of noncondensing bubbly jets by Sun and Faeth $(1985 \mathrm{a}, \mathrm{b})$ yielded similar results. The condensing jets involved nearly monodisperse carbon dioxide bubbles in water, injected vertically upward in still water. The carbon dioxide dissolves in the water while dissolved air comes out of solution and accumulates in the bubbles. Thus, the bubbles never entirely disappear but reach terminal diameters roughly $20 \%$ of their initial diameter. Mean and fluctuating phase velocities were measured using LDA; mean bubble number fluxes were measured using Mie scattering; and bubble diameters and number intensities were measured using flash photography. Bubble number intensity is defined as the number of bubbles along a cord-like path through the flow, per unit crossectional area of the path. This quantity can be related to the local void fraction of the flow and is easily computed from the analysis for

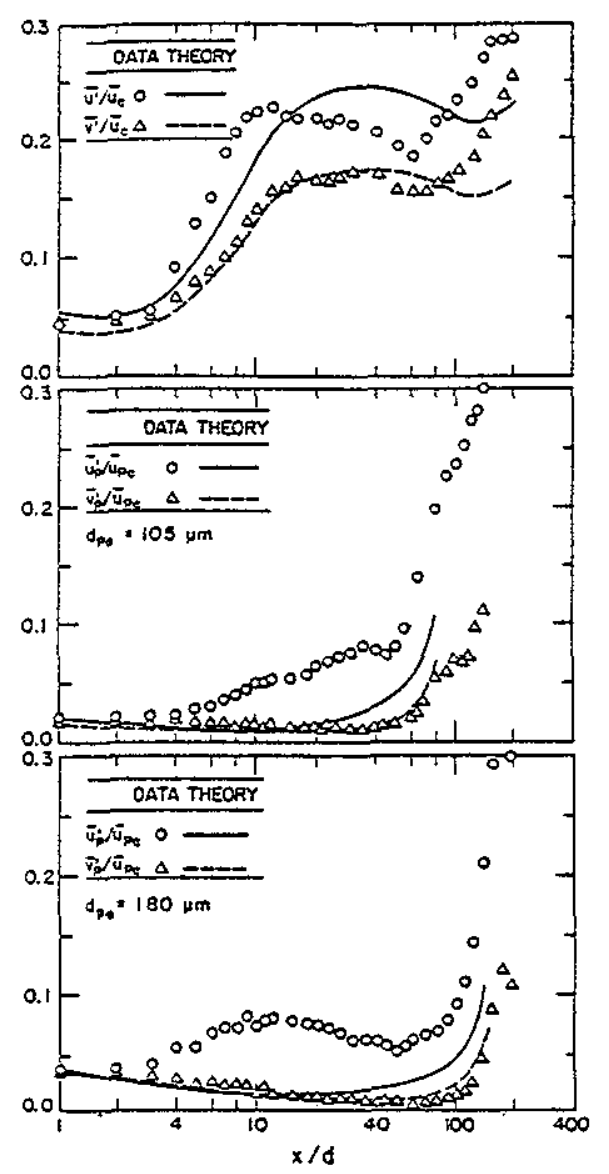

Fig. 14. Fluctuating phase velocities along the axis of round, ultra-dilute combusting sprays. From Shuen et al. (1985b).

companson with measurements. The advantage of bubble number intensity is that it is less subject to error than its deconvolution to give local void fractions (Sun and Faeth, 1985a).

Predictions and measurements of bubbie number intensity for a condensing bubbly jet are illustrated in Fig. 15. Predictions of the LHF, DSF, SSF and SSF-TM analyses are shown, the last including effects of turbulence modulation with $\mathrm{C}_{\varepsilon 3}=0.015$. The overall results are similar to Fig. 2 for the particle-laden jet of Yuu et al. (1978). In general, the LHF and DSF methods over- and underestimated the extent of the bubble containing region. In this case, however, relative velocities are small near the injector and the LHF analysis provides fair predictions at the lowest axial station. The stochastic methods, however, provide reasonably good predictions of the transition from near-LHF conditions near the injector to the $x / d=60$ position where both finite interphase transport rates and turbulent dispersion are important. Allowing for turbulence modulation does not have an important effect on the results illustrated in Fig. 15. Including turbulence modulation increases levels of turbulence kinetic energy which slightly widens the predicted flow due to enhanced turbulent dispersion.

The measurements of particle number flux, illustrated in Fig. 16, are a more sensitive indictor of flow widths than bubble number intensities. In this case, the two separated flow models yield similar results near the jet exit. but turbulence modulation causes a much wider profile at the 


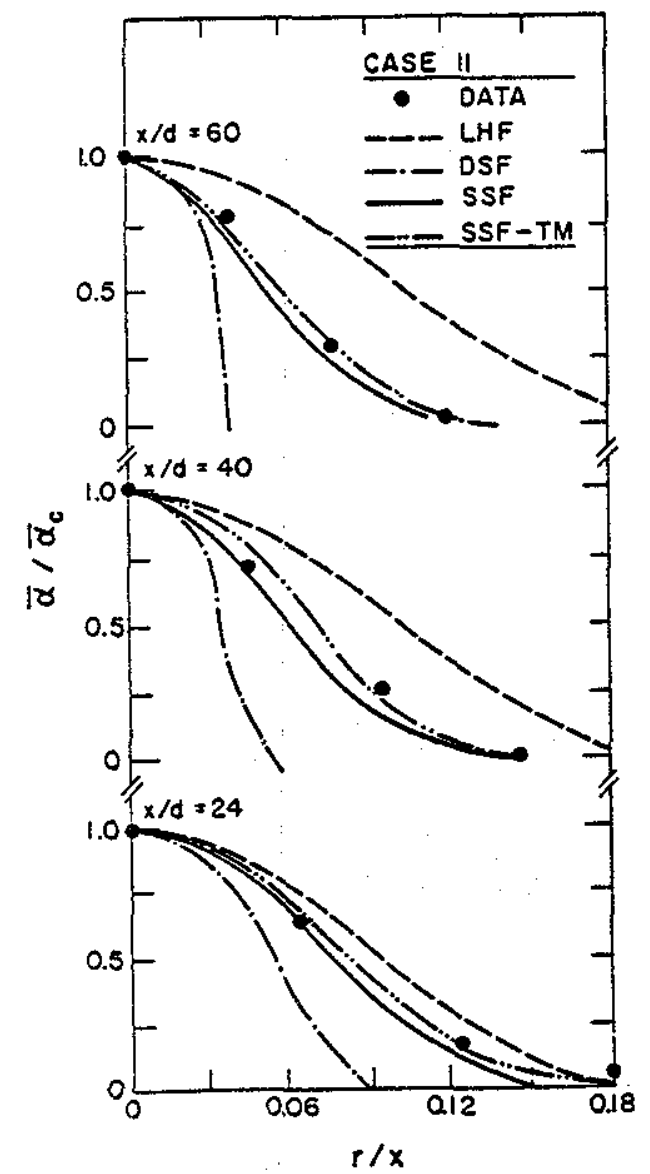

Fig. 15. Mean bubble number intensity distributions in a bubbly condensing jet. From Sun et al. $(1985 a, c)$.

$x / d=60$ position where the measurements are wider than both predictions. This is somewhat surprising since the flow becomes more dilute far from the injector and effects of turbulence modulation would be expected to decrease. The behavior is caused by relative velocities becoming comparable to mean liquid velocities at large $x / d$. The production of turbulence by the particle source term becomes comparable to turbulence production by shear forces in the continuous phase. This suggests that dilute flows having similar properties might be profitably studied to gain a better understanding of turbulence modulation than is possible in dense dispersed flows where experimental uncertainties are large.

Measurements and predictions of mean and fluctuating bubble velocities are illustrated in Fig. 17. Due to low liquid phase velocities for these test conditions, effects of relative velocities are significant. This can be seen by noting that the LFF predictions on the figure roughly correspond to mean liquid velocities. Mean radial bubble velocities increase monotonically with increasing radial distance, unlike their liquid-phase counterpart which reaches a maximum and then becomes negative near the edge of the flow due to entrainment of ambient fluid. This doesn't occur for the bubbles since no bubbles are entrained from the surroundings. Sprays and particle-laden jets have similar behavior. All the separated flow analyses provide reasonably good predictions of mean bubble velocities.

Bubble velocity fluctuations, pictured in the lower part of Fig. 17, exhibit significant levels of anisotropy. This behavior is not represented by the stochastic predictions,

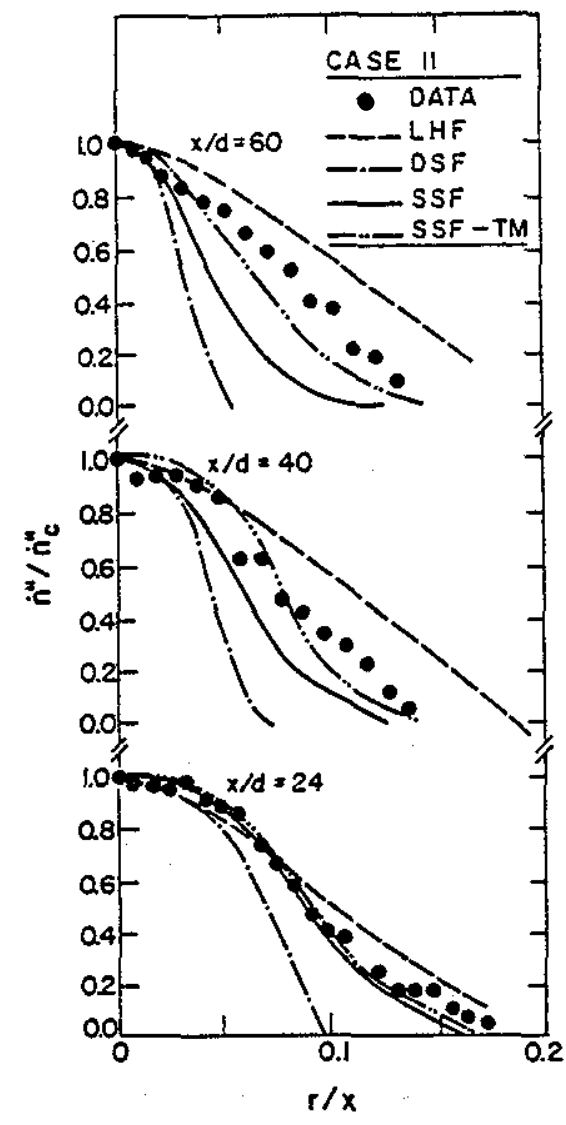

Fig. 16. Mean bubble number flux distributions in a round bubbly condensing jet. From Sun et al. (1985a).

which are nearly isotropic, which is quite different from the particle-laden jet discussed earlier. The reason for this is that bubbles adjust rapidly to local conditions in comparison to particles in gases. As a result, the assumption of anisotropic turbulence in the stochastic analyses causes streamwise fluctuations to be underestimated. Radial velocity fluctuations are slightly underestimated as well; this is partly attributed to variations of the initial distribution from the monodisperse distribution assumed in the computations (Sun et al., 1985a,c). Including effects of turbulence modulation causes a slight increase of bubble velocity fluctuations, but these differences are comparable to experimental uncertainties.

While consideration of turbulence modulation improved predictions of bubble spread rates slightly, predictions of rates of spread and turbulence levels in the continuous phase were overestimated as a result (Sun et al., $1985 \mathrm{c})$. This follows from the strong contribution of the bubble source term in the $k$ equation, which does not involve an empirical constant aside from neglecting the term entirely. The difficuity appears to involve the multiple turbulent length scales introduced by the bubble phase. Contributions of bubbles to turbulence occur at smaller scales than the energycontaining range of the continuous phase. Thus bubblegenerated turbulence enters the turbulent eddy cascade at small scales and dissipates more rapidly, tending to contribute less to turbulence properties important for mixing than is implied by the bubble source term in the $\mathrm{k}$ equation. Properly treating such effects requires more information on turbulent spectra in dilute dispersed flows. Multiple scales present substantial difficulties for current higher-order turbulence models; therefore, treating multiple-scale effects will not be trivial. 


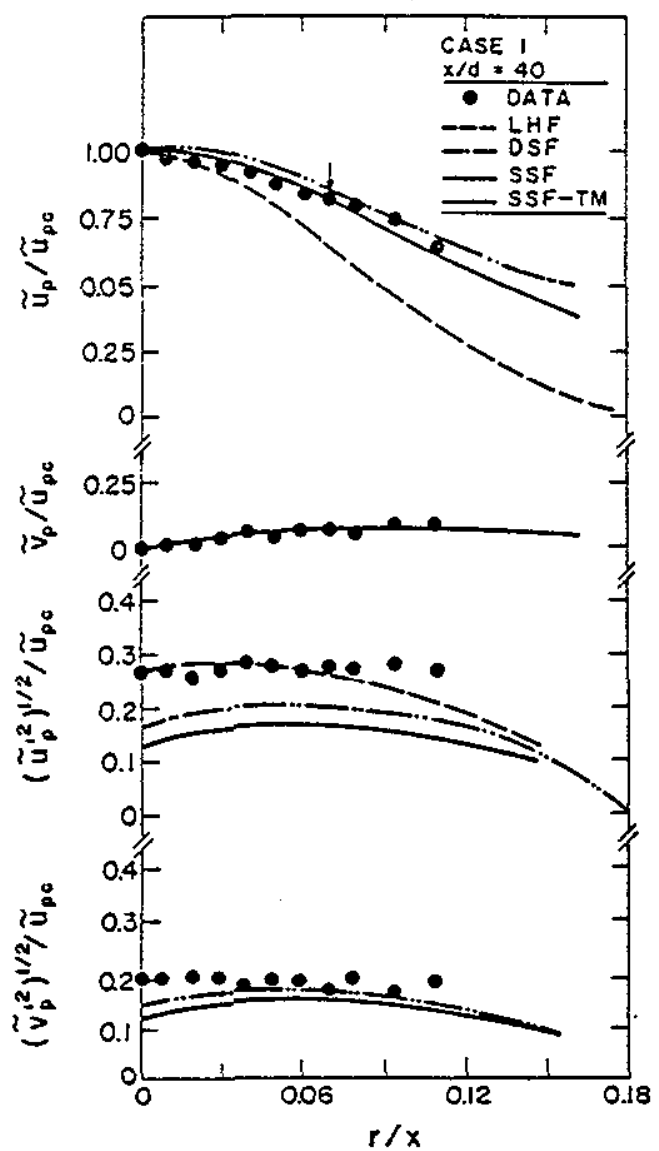

Fig. 17. Mean and fluctuating bubble velocities in a round bubbly condensing jet. From Sun et al. $(1985 a, c)$.

Viewing all the evidence presented here, it is clear that effects of turbulence modulation are important near regions of dense dispersed flows and in dilute flows dominated by effects of relative velocities between the phases. The stochastic method of treating turbulence modulation proposed by Shuen et al. (1985a) is tentative, empiricism in the $\varepsilon$ equation is not well established, and the method may have conceptual difficulties since it does not provide for effects of scale caused by differences between the size of dispersed phases and length scales of the continuous phase. Clearly, more work will be required to resolve these issues. Dilute dispersed flows dominated by slip are suggested for such studies, since they are experimentally accessible and highlight effects of turbulence modulation.

\section{Dense Dispersed Jets.}

Some aspects of dense dispersed jets have been considered thus far, based on observations near the boundaries of dilute dispersed flows. Additional information based on direct observations of dense sprays will be discussed in the following. Bracco $(1983,1985)$, Lefebvre (1980), Sirignano (1983) and Faeth (1983b) provide reviews treating aspects of this problem in more detail.

It is tempting to think of a dense spray as a closepacked collection of roughly spherical drops. Unfortunately, this picture is not accurate. A flash photograph of the flow at the exit of an air atomizing injec:or appears in Fig. 18. This spray was well-atomized, yielding

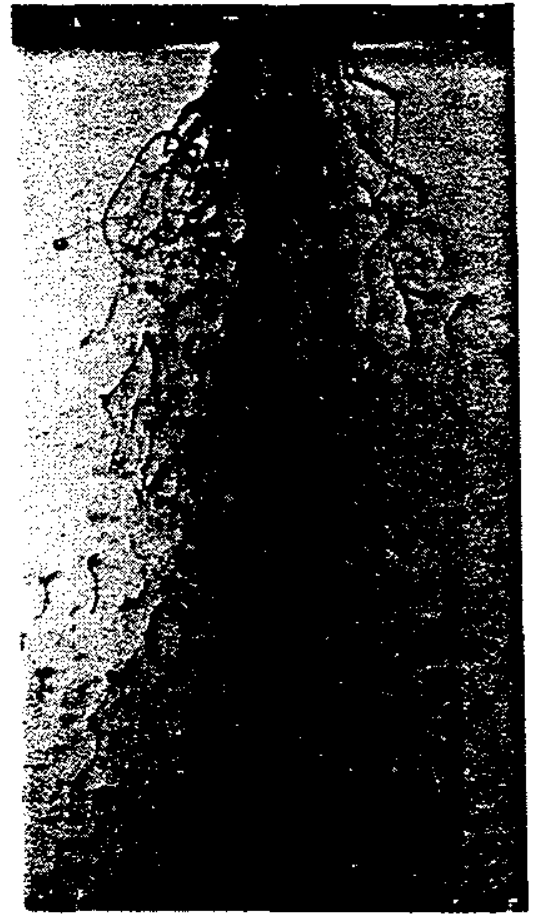

Fig. 18. Flash photograph of the flow at the exit of an air-atomizing injector.

an SMD of $30 \mu \mathrm{m}$ at $\mathrm{x} / \mathrm{d}=50$ (Solomon et al.; 1985a,b). Clearly, the flow involves dispersed-phase elements having complex shapes, ligaments, irregular drops, etc., which persist throughout the dense spray region. Similar observations near the exit of twin-fluid injectors abound (Lefebvre, 1980); therefore irregular liquid elements are an essential part of the dense spray region of such injectors.

Similar difficulties are encountered for pressureatomized sprays used in diesel and rocket engines. Fine drops are usually observed near the edge of the flow when operation is in the atomization region of pressure-atomizing injectors (Bracco, 1983, 1985; Wu et al., 1983), but direct observation of the interior dense spray region has not been possible. Hiroyasu et al. (1982), however, have obtained indirect evidence that a continuous liquid core extends far downstream of the injector exit. Bracco (1983), proposed the following semi-empirical expression for the length of this liquid core

$$
\mathrm{L}_{\mathrm{fd}} / \mathrm{d}=8\left(\rho_{\mathrm{f}} / \rho_{\infty}\right)^{1 / 2}
$$

This relationship suggests extensive core lengths, reaching several hundred injector diameters at low pressures. With a contiguous liquid core of this length, it seems likely that other large and irregular liquid elements are present in the dense portion of the flow as well. Thus, irregular liquid elements appear to be a ubiquitous aspect of all dense sprays.

Dispersed elements having irregular shapes pose serious conceptual difficulties for separated-flow-analysis of dense sprays. Furthermore, the characteristic times of breakup processes are comparable to residence times of the liquid in the dense region of the flow, but very little intormation is available to treat such processes. Findings discussed earlier suggest that collisions might play an 
important role in the unusual spread rates of the flow in the dense spray region of twin-fluid injectors - at least at low pressures. Analysis by O'Rourke and Bracco (1980) suggests that collisions strongly influence drop size distributions at the start of the dilute spray region of pressure atomized sprays as well.

In view of the complexities of these phenomena, prospects are not good for developing separated flow analysis of dense sprays in the near future. However, recent observations by Wu et al. (1983, 1984) suggest an alternative that could provide a better understanding of at least some dense spray phenomena. This involves use of the LHF approximation to model the mixing properties and void structure of the dense region of pressure atomized sprays. The LHF approximation has been used to study various aspects of diesel sprays, penetration, spray trajectories, etc., for some time (Faeth, 1977). Wu et al. (1983) find that this approach provides reasonable estimates of spray angles. Measurements of velocities in pressure-atomized sprays, using LDA, are also given as evidence that LHF ideas might be pertinent in dense sprays (Wu et al., 1984). These findings are less convincing, however, since only amplitude discrimination was used to distinguish phase velocities and such results are likely to be biased by the large number of small drops present in such flows.

Evidence suggesting that LHF analysis is deficient for sprays is widespread as well. Measurements discussed earlier indicate significant effects of relative velocities in almost every dilute spray where detailed measurements were made. Mao et al. (1981) also studied high pressure combusting pressure atomized sprays and found that LHF analysis overestimated rates of spray development, even at pressures approaching $100 \mathrm{~atm}$. Similar to all dense spray measurements, however, their experimental findings involved substantial uncertainties. Furthermore, their computations concerning effects of finite interphase transport rates ignored effects of drop shattering, which could be very important at high pressures when the stabilizing effect of surface tension decreases as drops approach their thermodynamic critical point (Faeth, 1983b). Finally, quantitative deficiencies aside, LHF analysis did provide correct trends of the effect of pressure on the measurements of Mao et al. (1981).

Clearly, work thus far has not successfully resolved the controversy concerning application of LFF analyses to the dense regions of pressure-atomized sprays. If the method is applicable for these flows it would be helpful for gaining a better understanding of dense sprays, with little empiricism beyond that needed for analysis of single-phase turbulent flows. Another advantage is that LHF analysis requires very little information concerning initial conditions of the analysis, offering good prospects for practical applications.

The large spread rates in the dense region of sprays from twin-fluid injectors at low pressures provides less encouragement for LHN analysis. The difficulties are apparent from the results illustrated in Figs. 6 and 9. However, more information is needed at pressure ranges of interest for combustion processes, and for other injector configurations, to see if there are conditions where the relatively simple LHF formalism could provide useful results.

\section{Conclusions}

Effects of finite interphase transport rates and turbulent dispersion were important in the dilute dispersed flows considered here; therefore, the LHF method, which ignores finite interphase transport rates and the DSF method, which ignores turbulent dispersion, were not very effective.
2. The SSF method, which treats both finite interphase transport rates and dispersed-phase/turbulence interactions using random walk computations for dispersed phase motion and transport, yielded encouraging results for the present dilute dispersed flows. Flows considered included particle-laden jets, nonevaporating sprays, evaporating sprays, combusting sprays, noncondensing bubbly jets and condensing bubbly jets, which represents a wide variety of phase interactions and fluid properties.

3. The conserved-scalar formalism and a relatively unsophisticated $\mathrm{k}-\varepsilon \cdot \mathrm{g}$ turbulence model were used to estimate continuous-phase properties during present computations concerning dilute dispersed flows. This approach should be extended to consider anisotropic velocity fluctuations and correlations between density (mixture fraction) and velocity fluctuations, since evidence was found that dispersed-phase/turbulence interactions were influenced by these properties.

4. Effects of turbulence modulation (modification of continuous-phase turbulence properties by the dispersed phase) were observed near regions of dense dispersed flow and in regions of dilute dispersed flows where relative velocities are comparable to continuous-phase velocities. Current methods of estimating effects of turbulence modulation are not well-developed and deserve further study. Properly treating differences in the scale of the energy-containing eddies of the continuous phase and turbulence scales introduced by the motion of the dispersed phase is a particular concern.

5. Existing information on combusting dilute dispersed flows is very limited and more measurements are needed. For the nonpremixed and ultra-dilute combusting sprays considered here, drops largely evaporated in regions where no oxidant was present. However, drops were observed to penetrate the flame zone; therefore, more information is needed concerning the initiation and stability of drop envelope flames for conditions representative of combusting sprays.

6. Dense sprays involve irregular liquid elements (ligaments, etc.) and significant effects of collisions and breakup. The complexities of these phenomena limit prospects for the development of detailed separated flow analyses of dense sprays in the near future. Recent work, however, suggests that LHF analysis might be effective in the dense-spray region of pressure atomized sprays, in spite of deficiencies noted earlier in dilute sprays. Additional measurements are needed to definitively evaluate this suggestion.

\section{Acknowledgements}

The author's research on multiphase transport processes was sponsored by the National Aeronautics and Space Administration, Grant No. NAG 3-190, under the technical management of $\mathrm{R}$. Tacina of the Lewis Research Center; the Office of Naval Research, Contract No. N00014 80-C-0517 and N00014-85-C-0148, under the technical management of R. D. Ryan and M. K. Ellingsworth; and the Air Force Office of Scientific Research, Grant No. AFOSR85-0244, under the technical management of J. Tishkoff. 


\section{REFERENCES}

Al Taweel, A.M. and Landau, J. (1977) Turbulence modulation in two-phase jets. Int.J. Multiphase Flow $3,341-351$.

Bilger, R.W. (1976) Turbulent jet diffusion flames. Prog. Energy Combust. Sci, 1, 87-109.

Bilger, R.W. (1977) Reaction rates in diffusion flames. Comb. Flame 30, 277-284.

Bracco, F.V. (1983) Structure of high-speed full-cone sprays. Recent Advances in Gas Dynamics (C. Casci, ed.), Plenum Publishing Corp., New York.

Bracco, F.V. (1985) Modeling of engine sprays. SAE Paper No. 850394.

Chigier, N.A. (1976) The atomization and burning of liquid fuel sprays. Prog, Energy Combust. Sci. 2, 97-114.

Clift, A.K., Grace, J.R. and Weber, M.E. (1978) Bubbles. Droos and Particles, Academic Press, New York, 185 . 319.

Crowe, C.T. (1982) Review-numerical methods for dilute gas-particle flows. I. Fluids Engr, 104, 197-203.

Crowe, C.T., Sharma, M.P. and Stock, D.E. (1977) The particle-source-in-cell (PSI-CELL) model for gas-droplet flows. I Fluids Engrg. 99, 325-332.

Csanady, G.T. (1963) Turbulent diffusion of heavy particles in the atmosphere. I. Atm. Sci. 20, 201.

Dukowicz, J.K. (1980) A particle-fluid numerical model for liquid sprays. I.Comp. Phys. 35, 229-253.

Elgohbashi, S.E. and Abou-Arab, T.W. (1983) A twoequation turbulence model for two-phase flows. Phys. Eluids 26, 931-398.

Faeth, G.M. (1977) Current status of droplet and liquid combustion. Prog Energy Combust. Sci. 3, 191-224.

Faeth, G.M. (1983a) Recent advances in modeling particle transport properties and dispersion in turbulent flow. Proceedings of the ASME.JSME Thermal Engineering Joint Conference, Vol. II, ASME, New York, 517-534.

Faeth, G.M. (19836) Evaporation and combustion of sprays. Brog Energy Combust. Sci, 9, 1-76.

Faeth, G.M. and Samuelson, G.S. (1985) Fast reaction non premixed combustion. Evaluation of Data on Simple Turbulent Elows (W.C. Strahle and S.G. Lekoudis, ed.), AFOSR TR-85 0880.

Goldschmidt, V.W., Housholder, M.K., Ahmadi, G. and Chuang, S.C. (1972) Turbulent disperison of small particles suspended in turbulent jets. Brogress in Heat and Mass Transfer (G. Hetsroni, S. Sideman and J.P. Hartnett, ed.), Vol. 6, Pergammon Press, Oxford, 487. 508.

Gosman, A.D. and Ioannides, E. (1981) Aspects of computer simulation of liquid-fueled combustion. AlAA Paper No. 81-0323.

Hinze, J.O. (1972) Turbulent fluid and particle interaction. Prog, Heat Mass Trans. 6, 433-452.
Hinze, J.O. (1975) Turbulence, 2nd Ed., McGraw-Hill, New York, 427 and 724-734.

Hiroyasu, H. Shimizu, M. and Arai, M. (1982) The breakup of a high speed jet in a high temperature gaseous atmosphere. Proceedings of the 2nd International Conference on Liquid Atomization and Spray System, Madison, WI.

Hjelmfelt, A.T. and Mockros, L.F. (1966) Motion of discrete particles in a turbulent field. App. Sci Res. 6, 149.

Jeng, S.-M., Chen, L.-D. and Faeth, G.M. (1982) The structure of buoyant methane and propane diffusion flames. Nineteenth Symposium (International) on Combustion, The Combustion Institute, Pittsburgh, $1077+1085$

Jeng, S.-M. and Faeth, G.M. (1984a) Species concentrations and turbulence properties in buoyant methane diffusion flames. L Heat Transfer 106, 721-727.

Jeng, S.-M. and Faeth, G.M. (1984b) Predictions of mean scalar properties in turbulent propane diffusion flames. J.Heat Trans 106, 891-893.

Jurewicz, J.T. and Stock, D.E. (1976) A numerical model for turbulent diffusion in gas-particle flows. ASME Paper 76-WA-FE-33.

Laats, M.K. and Frishman, F.A. (1970a) Assumptions used in calculating the two-phase jet. Euid Dynamics 5 , 333-338.

Laats, M.K. and Frishman, F.A. (1970b) Scattering of an inert admixture of different grain size in a two-phase axisymmetric jet. Heat Transfer Soviet Res, 2, 7-12.

Law, C.K. (1982) Recent advances in droplet vaporization and combustion. Prog Eneroy Combust. Sci. 8, 171201.

Lefebvre, A.H. (1980) Airblast atomization. Prog Energy Combust. Sci, 6, 233-261.

Levy, Y. and Lockwood, F.C. (1981) Velocity measurements in a particle-laden turbulent free jet Comb Elame 40, 333-339.

Liew, S.K., Bray, K.N.C. and Moss, J.B. (1981) A flamelet model of turbulent non-premixed combustion. Comb. Sci. Tech 27, 69-73.

Liew, S.K., Bray, K.N.C. and Moss, J.B. (1984) A stretched laminar flamelet model of turbulent nonpremixed combustion. Comb. Flame 56, 199-213.

Lockwood, F.C. and Naguib, A.S. (1975) The prediction of fluctuations in the properties of free, round-jet, turbulent, diffusion flames. Comb. Flame 24, 109-124.

Mao, C.-P., Szekely, G.A., Jr. and Faeth, G.M. (1980) Evaluation of a locally homogeneous flow model of spray combustion. I. Energy 4, 78-87.

Mao, C.-P., Wakamatsu, Y. and Faeth, G.M. (1981) A simplified model of high pressure spray combustion. Eighteenth Symposium (Intemational) on Combustion. The Combustion Institute, Pittsburgh, 337-347. 
McComb, W.D. and Salih, S.M. (1977) Measurements of normalized radial concentration profiles in a turbulent aerosol jet using a laser-Doppler anemometer. I. Aerosol Sci 8, 171-181.

McComb, W.D. and Salih, S.M. (1978) Comparison of some theoretical concentration profiles for solid particles in a turbulent jet with the results of measurements using a laser-Doppler anemometer. J.Aerosel_Sci, 9, 299313.

Modarress, D., Tan, H. and Elghobashi, S. (1984) Twocomponent LDA measurement in a two-phase turbulent jet. AIAAJ 22, 624-630.

Moore, D.W. (1965) The velocity of rise of distorted gas bubbles in a liquid of small viscosity. LEluid.Mech. $23,749-766$.

Odar, F. and Hamilton, W.S. (1964) Force on a sphere accelerating in a viscous fluid. L.Eluid Mech. 18, 302314.

O'Rourke, P.J. and Bracco, F.V. (1980) Modeling of drop interactions in thick sprays and a comparison with experiments. Inst. Mech. Engr. Pub. ISBN 0 $852984693,101-116$.

Peskin, R.L. (1975) Some fundamental research problems in gas-solid flows. Air: I. Pollution Control and Clean Energy, AIChE Symposium Series 147, Vol. 71, 52-59.

Sirignano, W.A. (1983) Fuel droplet combustion and spray combustion theory. Brog Energy Combust. Sci. 9, 291-322

Shearer, A.J. Tamura, H. and Faeth, G.M. (1979) Evaluation of a locally homogeneous flow model of spray evaporation. J. Energy 3, 271-278.

Shuen, J.-S., Chen, L.-D. and Faeth, G.M. (1983a) Evaluation of a stochastic model of particle dispersion in a urbulent round jet. AIChE J. 29, 167-170.

Shuen, J.-S. Chen, L.-D. and Faeth, G.M. (1983b) Predictions of the structure of turbulent particle-laden round jets. AIAA $21,1483-1484$.

Shuen, J.-S., Solomon, A.S.P., Zhang, Q.-F. and Faeth, G.M. (1985a) Structure of particle-laden jets: predictions and measurements. ALAAJ 23, 396-404.

Shuen, J.-S., Solomon, A.S.P. and Faeth, G.M. (1985b) Drop-turbulence interactions in a diffusion flame. AIAA I in press.

Snyder, W.H. and Lumley, J.L. (1971) Some measurements of particle velocity autocorrelation functions in a turbulent flow. LEluid.Mech. 48, 41-71.

Solomon, A.S.P., Shuen, J.-S. Zhang, Q.-F. and Faeth, G.M. (1985a) Structure of nonevaporating sprays: I. near-injector conditions and mean properties. AIAA J. $23,1548-1555$.

Solomon, A.S.P., Shuen, J.-S., Zhang, Q.-F. and Faeth. G.M. (1985b) Structure of nonevaporating sprays: T? drop and turbulent properties. AlAÁ 23, 1724-1731;.
Solomon, A.S.P., Shuen, J.-S. Zhang, Q.-F. and Faeth, G.M. (1985c) Measurements and predictions of the structure of evaporating sprays. L. Heat Trans. 107, 679-686.

Soo, S.L. (1967) Eluid Dynamics of Multiphase Systems, Blaisdel Publishing Co., Waltham, MA.

Sun, T.-Y. and Faeth, G.M. (1985a) Structure of turbulent bubbly jets - I. methods and centerline properties. Int. L.Multiphase Elow, in press.

Sun, T.-Y. and Faeth, G.M. (1985b) Structure of turbulent bubbly jets -- II. phase property profiles. Int. I. Multiphase Elow, in press.

Sun, T.-Y., Parthsarathy, R.N. and Faeth, G.M. (1985a) Structure of bubbly round condensing jets. Multiphase Elow and Heat Transfer (V.X. Dhir, J.C. Chen and O.C. Jones, ed.) HTD-Vol. 47, ASME, New York, 7584.

Sun, T.-Y., Parthasarathy, R.N. and Faeth, G.M. (1985b) Bubbly condensing jets -. I. methods and near-source properties. L. Heat Transfer, submitted.

Sun, T.-Y., Parthasarathy, T.N. and Faeth, G.M. (1985c) Bubbly condensing jets -. II. mean and turbulent structure. I. Heat Transfer, submitted.

Szekely, G.A., Jr. and Faeth, G.M. (1983) Effects of envelope flames on drop gasification rates in turbulent diffusion flames. Comb. Elame 49, 255-259.

Tchen, C.M. (1947) Mean value and correlation problems connected with the motion of small particles suspended in a turbulent fluid. Ph.D. Thesis, University of Delft, The Hague.

Williams, F.A. (1985) Some theoretical aspects of spray combustion. ASME Paper No. 85-WA/HT-44.

Wu, K.-J., Su, C.-C. Steinberger, R.L., Santavicca, D.A. and Bracco, F.V. (1983) Measurements of spray angle of atomizing jets. LEluids Engr, 105, 406-415.

Wu, K..J. Santavicea, D.A., Bracco, F.V. and Coghe, A. (1984) LDV measurements of drop velocity in dieseltype sprays. ALAAJ, 22, 1263-1270.

Wygnanski, I. and Fiedler, H.E. (1969) Some measurements in the self-preserving jet. J. Fluid Mech. $38,577-612$.

Yudine, M.I. (1959) Physical considerations on heavy particle diffusion. Adv. Geophys. 6, 185-191.

Yuu, S., Yasukouchi, N., Hirosawa, Y. and Jotaki, 1. (1978) Particle turbulent diffusion in a dust laden round flame. AIChE J 24, 509-195.

Zhang, Q.-F., Shuen, J.-S., Solomon, A.S.P. and Faeth, G.M. (1985) Structure of ducted particle-laden jers. AIAA I 23, 1123-1125. 